\title{
THE NUMERICAL INTERFACE COUPLING OF NONLINEAR HYPERBOLIC SYSTEMS OF CONSERVATION LAWS: II. THE CASE OF SYSTEMS
}

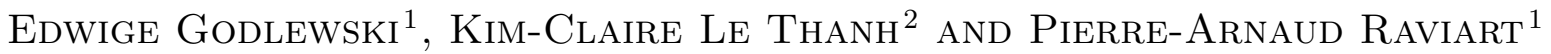

\begin{abstract}
We study the theoretical and numerical coupling of two hyperbolic systems of conservation laws at a fixed interface. As already proven in the scalar case, the coupling preserves in a weak sense the continuity of the solution at the interface without imposing the overall conservativity of the coupled model. We develop a detailed analysis of the coupling in the linear case. In the nonlinear case, we either use a linearized approach or a coupling method based on the solution of a Riemann problem. We discuss both approaches in the case of the coupling of two fluid models at a material contact discontinuity, the models being the usual gas dynamics equations with different equations of state. We also study the coupling of two-temperature plasma fluid models and illustrate the approach by numerical simulations.
\end{abstract}

Mathematics Subject Classification. 35L50, 35L65, 65M12, 65M30, 65-04, 76M12.

Received: April 2, 2004. Revised: November 16, 2004.

\section{INTRODUCTION}

In the modeling of complex problems, different mathematical models are frequently used in different regions of interest. On the one hand, one can assume that some physical effects are negligible in some domains, which amounts to drop the corresponding terms in the equations of the complete model. For instance, when "small" relaxation times occur, one can often replace the model by its relaxation approximation where some equilibrium is assumed. On the other hand, one can suppose that the phenomenon is fully three-dimensional in some domains and only one or two-dimensional in other ones. Mathematically, this leads to couple different (nonlinear) systems of partial differential equations of different sizes at various artificial boundaries. The study of such coupling is of rapidly increasing importance in engineering problems where one wants to take into account the physical complexity of a phenomenon but at a reasonable computing cost. However, the mathematical and numerical analyses of this coupling lead to nonconventional and highly challenging problems which have been very little investigated.

The purpose of this series of papers is to contribute to the study of the coupling of nonlinear hyperbolic systems from both mathematical and numerical points of view. The coupling condition (Condition (2.6)) results by expressing that two boundary value problems should be well-posed, which resumes to impose as far as possible the continuity of the solution at the interface. In a first paper [17], we have considered the scalar case

\footnotetext{
Keywords and phrases. Conservation laws, Riemann problem, boundary value problems, interface coupling, finite volume schemes.

${ }^{1}$ Laboratoire Jacques-Louis Lions, Université Pierre et Marie Curie, 75252 Paris Cedex 05, France. godlewski@ann.jussieu.fr

2 CEA, BP 12, 91680 Bruyères le Chatel, France. kim-claire.le-thanh@cea.fr
} 
where we couple two different one-dimensional conservation laws at a fixed interface. In this paper, we begin the study of the coupling of systems. Although we restrict ourselves to the one-dimensional case and to systems of the same size, the situation is still far more complicated than in the scalar case. As expected, we are able to give a detailed analysis of the coupling only in the linear case. In the nonlinear case, one can either use a linearized approach which gives a heuristic answer or a coupling method based on the solution of a Riemann problem. We will discuss both approaches in the case of the coupling of two fluid models at a material contact discontinuity, the models being the usual gas dynamics equations in Lagrangian coordinates, with different equations of state (the numerical approach of this problem is considered in [18], and in $[1,11]$ for Eulerian coordinates). We will also study the coupling of two-temperature fluid models for a quasi-neutral ionized plasma with different current densities but the same equation of state (see [26] for a detailed discussion of these models and their coupling in the context of a physical application). Though in this 4-equation system, the flux function of only one equation changes when crossing the interface, the coupling already presents significative difficulties, such as the non uniqueness for the coupled problem and the possible occurrence of resonance.

The plan of the paper is as follows. In Section 2, we introduce the coupling problem at an interface of two nonlinear systems of conservation laws and detail the coupling constraint. We also introduce the associated numerical coupling procedure. In Section 3, we consider the linear case with constant coefficients and study the well-posedness of the coupled Cauchy problem. Indeed, depending on the number of entering or outgoing characteristic lines on each side of the interface, this problem may be ill-posed in the sense that it possesses a continuum of solutions. In Section 4, we apply the previous results to the linearized Euler system of gas dynamics. In Section 5, we study the coupling of various standard fluid models at a material contact discontinuity whose position is kept fixed when working in Lagrangian coordinates. The next sections are devoted to the coupling of plasma models. In Section 6, we introduce the two-temperature plasma model where the current density plays the role of a parameter and we solve the associated Riemann problem. In Section 7, we consider the coupling of two plasma models corresponding to different densities. We prove that, in physically relevant situations, the coupled Riemann problem admits a continuum of solutions depending on a one-dimensional parameter. In Section 8, we solve numerically this coupled Riemann problem and we check that the obtained approximate solution depends only slightly on the chosen numerical scheme and on the CFL. We also study the influence of initial data and of the equation of state on the numerical solution.

\section{The COUPLING PROBLEM FOR SYSTEMS}

Let $\Omega \subset \mathbb{R}^{p}$ be the set of states and let $\mathbf{f}_{\alpha}, \alpha=L, R$, be two "smooth" functions from $\Omega$ into $\mathbb{R}^{p}$. Given a function $\mathbf{u}_{0}: x \in \mathbb{R} \rightarrow \mathbf{u}_{0}(x)$, we want to find a function $\mathbf{u}:(x, t) \in \mathbb{R} \times \mathbb{R}_{+} \rightarrow \mathbf{u}(x, t) \in \Omega$ solution of

$$
\begin{aligned}
& \frac{\partial \mathbf{u}}{\partial t}+\frac{\partial}{\partial x} \mathbf{f}_{L}(\mathbf{u})=\mathbf{0}, \quad x<0, t>0 \\
& \frac{\partial \mathbf{u}}{\partial t}+\frac{\partial}{\partial x} \mathbf{f}_{R}(\mathbf{u})=\mathbf{0}, \quad x>0, t>0
\end{aligned}
$$

and satisfying the initial condition

$$
\mathbf{u}(x, 0)=\mathbf{u}_{0}(x), \quad x \in \mathbb{R} .
$$

At the interface $x=0$, we need to supplement equations (2.1)-(2.3) with coupling conditions in order to obtain a well-posed problem. At that stage, we have first to define what we mean by an admissible boundary condition at $x=0$ for both systems (2.1) and (2.2). In fact we will assume in all the sequel that the systems (2.1) and (2.2) are hyperbolic, i.e. for $\alpha=L, R$, the Jacobian matrix $A_{\alpha}(\mathbf{u}) \equiv \mathbf{f}_{\alpha}^{\prime}(\mathbf{u})$ of $\mathbf{f}_{\alpha}(\mathbf{u})$ is diagonizable with real eigenvalues $\lambda_{\alpha, k}(\mathbf{u})$ and corresponding eigenvectors $\mathbf{r}_{\alpha, k}(\mathbf{u}), 1 \leq k \leq p$. Then we introduce the solution

$$
\mathbf{w}(x, t)=\mathbf{W}_{\alpha}\left(x / t ; \mathbf{u}_{L}, \mathbf{u}_{R}\right)
$$


of the Riemann problem

$$
\left\{\begin{array}{l}
\frac{\partial}{\partial t} \mathbf{w}+\frac{\partial}{\partial x} \mathbf{f}_{\alpha}(\mathbf{w})=\mathbf{0}, \quad x \in \mathbb{R}, t>0 \\
\mathbf{w}(x, 0)= \begin{cases}\mathbf{u}_{L}, & x<0 \\
\mathbf{u}_{R}, & x>0\end{cases}
\end{array}\right.
$$

We set for all $\mathbf{b} \in \Omega$,

$$
\left\{\begin{array}{l}
\mathcal{O}_{L}(\mathbf{b})=\left\{\mathbf{w}=\mathbf{W}_{L}\left(0-; \mathbf{u}_{L}, \mathbf{b}\right) ; \mathbf{u}_{L} \in \Omega\right\} \\
\mathcal{O}_{R}(\mathbf{b})=\left\{\mathbf{w}=\mathbf{W}_{R}\left(0+; \mathbf{b}, \mathbf{u}_{R}\right) ; \mathbf{u}_{R} \in \Omega\right\}
\end{array}\right.
$$

and following $[10,17]$, we define conditions of the form

$$
\mathbf{u}(0-, t) \in \mathcal{O}_{L}(\mathbf{b}(t)), \quad t>0
$$

and

$$
\mathbf{u}(0+, t) \in \mathcal{O}_{R}(\mathbf{b}(t)), \quad t>0,
$$

as admissible boundary conditions for (2.1) and (2.2) respectively. Hence natural coupling conditions for problem (2.1)-(2.3) consist in requiring

$$
\left\{\begin{array}{l}
\mathbf{u}(0-, t) \in \mathcal{O}_{L}(\mathbf{u}(0+, t)) \\
\mathbf{u}(0+, t) \in \mathcal{O}_{R}(\mathbf{u}(0-, t))
\end{array}\right.
$$

Other more rigorous ways of writing boundary conditions can be found in $[13,14,32]$, however using the formulation with Riemann problems is more practical and in the first paper of this series [17] devoted to the scalar case, we have shown that this was indeed a "reasonable" way of coupling two hyperbolic equations.

Remark 1. Note that the coupling conditions (2.6) do not imply the continuity of the flux at the interface: we have in general

$$
\mathbf{f}_{L}(\mathbf{u}(0-, t)) \neq \mathbf{f}_{R}(\mathbf{u}(0+, t))
$$

so that our coupling method is not conservative. However, there exist other approaches which are conservative. For instance we might want to write $(2.1)(2.2)$ in the form of the single system

$$
\frac{\partial \mathbf{u}}{\partial t}+\frac{\partial}{\partial x} \mathbf{f}(\mathbf{u}, x)=\mathbf{0}, x \in \mathbb{R}, t>0,
$$

with a flux function depending discontinuously on $x$,

$$
\mathbf{f}(\mathbf{u}, x)= \begin{cases}\mathbf{f}_{L}(\mathbf{u}), & x<0 \\ \mathbf{f}_{R}(\mathbf{u}), & x>0\end{cases}
$$

Such systems are studied for instance by Lyons [27], Klausen and Risebro [23], Towers [22,33], Adimurthi and Veerappa Gowda [2], Bale et al. [4], Seguin and Vovelle [31], Mishra [28] in the context of flow in porous media with discontinuous permeability, gravitational waves (sedimentation) or traffic flow (more references can be found in these papers). Since they are in conservative form, the Rankine-Hugoniot jump condition gives at $x=0$ the continuity of the flux as interface condition

$$
\left.\mathbf{f}_{R}(\mathbf{u}(0+, t))=\mathbf{f}_{L}(\mathbf{u})(0-, t)\right)
$$

in place of (2.6). Coming back to the nonconservative approach, we have

$$
\frac{\partial \mathbf{u}}{\partial t}+\frac{\partial}{\partial x} \mathbf{f}(\mathbf{u}, x)=\mathcal{M}, x \in \mathbb{R}, t>0,
$$

where $\mathcal{M}$ is a Dirac measure concentrated on $x=0$, with weight $\mathbf{f}_{R}(\mathbf{u}(0+, t))-\mathbf{f}_{L}(\mathbf{u}(0-, t))$. Conservation laws with such Dirac source terms are considered in $[9,19]$. 
Remark 2. Another way of satisfying the flux coupling condition (2.7) consists in writing (2.1) (2.2) in the form of an augmented system

with a Riemann datum for $a$

$$
\left\{\begin{array}{l}
\frac{\partial \mathbf{u}}{\partial t}+\frac{\partial}{\partial x} \mathbf{f}(\mathbf{u}, a)=\mathbf{0}, \\
\frac{\partial a}{\partial t}=0,
\end{array} \quad x \in \mathbb{R}, t>0,\right.
$$

$$
a(x, 0)=\left\{\begin{array}{l}
a_{L}, x<0, \\
a_{R}, x>0,
\end{array}\right.
$$

so that $a(x, t)=a(x, 0)$ is also piecewise constant, and a flux function $\mathbf{f}(\mathbf{u}, a)$ such that

$$
\mathbf{f}\left(\mathbf{u}, a_{L}\right)=\mathbf{f}_{L}(\mathbf{u}), \mathbf{f}\left(\mathbf{u}, a_{R}\right)=\mathbf{f}_{R}(\mathbf{u}) .
$$

Observe that the above system may be resonant at states $\left(\mathbf{u}_{*}, a_{*}\right)$ such that the (partial) Jacobian matrix $D_{\mathbf{u}} \mathbf{f}\left(\mathbf{u}_{*}, a_{*}\right)$ has a zero eigenvalue. Such problems have been studied by Isaacson and Temple [21].

Another type of augmented system is proposed in [18] in the case of material interfaces: they set $\mathbf{f}(\mathbf{u}, a)=(1-a) \mathbf{f}_{L}(\mathbf{u})+a \mathbf{f}_{R}(\mathbf{u})$ but the switching parameter $a$ satisfies $a_{t}+k(a)_{x}=0$ for some concave function $k$ such that $k(0)=k(1)=0$, so that the corresponding shock waves between 0 and 1 are stationary.

In these conservative approaches, one is faced in general with the non uniqueness of the solution for the Cauchy problem and thus of selecting an "admissible" solution; various selection criteria are proposed in the above references. Note that in the scalar case and for the first conservative approach, entropy conditions and uniqueness results have been recently proved by Audusse and Perthame [3].

In our coupled method, the problem of non uniqueness also arises; we have already met it in the scalar case (see [17]) when the signs of $f_{L}^{\prime}$ and $f_{R}^{\prime}$ change when crossing the interface, we may have an infinite number of solutions. We will meet it again in the next sections. However, we have not yet found out a satisfying criterium for selecting an "admissible" solution. On the other hand, resonance may be avoided at least in some cases as we will notice it in Section 7.

Let us turn to the numerical approximation of problem (2.1)-(2.3) and (2.6). We introduce a uniform mesh space $\Delta x$ and a time step $\Delta t$ and we set

$$
\mu=\frac{\Delta t}{\Delta x}, \quad x_{j+1 / 2}=\left(j+\frac{1}{2}\right) \Delta x, j \in \mathbb{Z}, \quad t_{n}=n \Delta t, n \in \mathbb{N} .
$$

Then, for $\alpha=L, R$, we are given a numerical flux function $\mathbf{g}_{\alpha}: \Omega^{2} \rightarrow \mathbb{R}^{p}$ consistent with the flux function $\mathbf{f}_{\alpha}$ and we consider the three-point numerical schemes

$$
\begin{cases}\mathbf{u}_{j-1 / 2}^{n+1}=\mathbf{u}_{j-1 / 2}^{n}-\mu\left(\mathbf{g}_{L, j}^{n}-\mathbf{g}_{L, j-1}^{n}\right), & j \leq 0, \\ \mathbf{u}_{j+1 / 2}^{n+1}=\mathbf{u}_{j+1 / 2}^{n}-\mu\left(\mathbf{g}_{R, j+1}^{n}-\mathbf{g}_{R, j}^{n}\right), & j \geq 0,\end{cases}
$$

where

$$
\mathbf{g}_{\alpha, j}^{n}=\mathbf{g}_{\alpha}\left(\mathbf{u}_{j-1 / 2}^{n}, \mathbf{u}_{j+1 / 2}^{n}\right), \quad \alpha=L, R,
$$

and $\mathbf{u}_{j+1 / 2}^{0}$ is an approximation of " $\mathbf{u}_{0}\left(x_{j+1 / 2}\right)$ ", for instance

$$
\mathbf{u}_{j+1 / 2}^{0}=\frac{1}{\Delta x} \int_{x_{j}}^{x_{j+1}} \mathbf{u}_{0}(x) \mathrm{d} x, \quad j \in \mathbb{Z} .
$$

The coupling of the difference schemes (2.8) is performed through the evaluation of $\mathbf{g}_{\alpha, 0}^{n}=\mathbf{g}_{\alpha}\left(\mathbf{u}_{-1 / 2}^{n}, \mathbf{u}_{1 / 2}^{n}\right)$, $\alpha=L, R$. We have proven in the scalar case that in a number of significant situations, the coupled numerical scheme "converges" to a solution of the coupled problem satisfying condition (2.6) (we refer to [17] for details).

Since in general $\mathbf{g}_{L, 0}^{n} \neq \mathbf{g}_{R, 0}^{n}$, the overall numerical scheme (2.8) is not conservative. However we can "enforce" the conservativity as explained in Remark 4. 
Remark 3. In order to obtain rather general theoretical results, we have made the implicit assumption that both numerical fluxes can be defined at the interface i.e., $\mathbf{g}_{\alpha, 0}^{n}=\mathbf{g}_{\alpha}\left(\mathbf{u}_{-1 / 2}^{n}, \mathbf{u}_{1 / 2}^{n}\right), \alpha=L, R$ make sense. However, there are situations where these formulae cannot be used. An example is given by [5] with a gas-liquid (airwater) compressible flow model, using a stiffened equation of state, which allows the pressure in the water to be negative (this is the cavitation phenomena). Though the boundary value problems are well-posed, a numerical problem arises if the values of the pressure on each side of the interface are not in agreement (negative in the liquid, positive in the gas). The numerical method must then be adapted.

Remark 4. Note that the coupled problem $(2.1)-(2.3),(2.6)$ is not in conservation form since, at $x=0$, the fluxes $\mathbf{f}_{L}(\mathbf{u}(0-, t))$ and $\mathbf{f}_{R}(\mathbf{u}(0+, t))$ differ in general. In fact we can enforce conservativity (which is indeed required in some physical problems) in the following way. Since the flux functions $\mathbf{f}_{\alpha}$ are defined up to an additive function of time, letting for instance $\mathbf{f}_{L}$ remain fixed, we replace in $(2.2) \mathbf{f}_{R}$ by

$$
\tilde{\mathbf{f}}_{R}(t, \mathbf{u})=\mathbf{f}_{R}(\mathbf{u})-\varphi(t),
$$

where the function $\varphi$ is determined in such a way that

$$
\tilde{\mathbf{f}}_{R}(t, \mathbf{u}(0+, t))=\mathbf{f}_{L}(\mathbf{u}(0-, t))
$$

i.e.,

$$
\varphi(t)=\mathbf{f}_{R}(t, \mathbf{u}(0+, t))-\mathbf{f}_{L}(\mathbf{u}(0-, t)) .
$$

At the numerical level, we obtain a conservative scheme by replacing in $(2.8) \mathbf{g}_{R, j}^{n}, j \geq 0$, by

$$
\tilde{\mathbf{g}}_{R, j}^{n}=\mathbf{g}_{R, j}^{n}-\varphi^{n}, \varphi^{n}=\mathbf{g}_{R, 0}^{n}-\mathbf{g}_{L, 0}^{n} .
$$

Observe that this does not change the numerical method except at infinity, or in practice at the artificial right boundary, where the numerical flux is modified as above. However this modification is somewhat arbitrary and should not be used in any context.

\section{The CASE OF LineAR SYSTEMS With CONSTANT COEFFICIENTS}

We analyze in this section the simplest possible case where

$$
\mathbf{f}_{\alpha}(\mathbf{u})=\mathbf{A}_{\alpha} \mathbf{u}, \alpha=L, R,
$$

where $\mathbf{A}_{L}$ and $\mathbf{A}_{R}$ are constant $p \times p$ matrices. As a preliminary step, we recall some standard results (cf. [16] for instance) concerning the initial boundary-value problem

$$
\left\{\begin{array}{l}
\frac{\partial \mathbf{u}}{\partial t}+\mathbf{A} \frac{\partial \mathbf{u}}{\partial x}=\mathbf{0}, \quad x>0, t>0 \\
\mathbf{u}(x, 0)=\mathbf{u}_{0}(x), x>0 \\
\mathbf{u}(0+, t) \in \mathcal{O}_{R}(\mathbf{b}(t)), t>0
\end{array}\right.
$$

where for all $\mathbf{b} \in \mathbb{R}^{p}$

$$
\mathcal{O}_{R}(\mathbf{b})=\left\{\mathbf{w}=\mathbf{W}(0+; \mathbf{b}, \mathbf{v}) ; \mathbf{v} \in \mathbb{R}^{p}\right\}
$$

We suppose that the eigenvalues $\lambda_{k}$ of the matrix $\mathbf{A}$ are real, distinct and ordered as

$$
\lambda_{1}<\lambda_{2}<\cdots<\lambda_{q} \leq 0<\lambda_{q+1}<\cdots<\lambda_{p}
$$

We denote by $\mathbf{r}_{k}$ and $\ell_{k}$ the corresponding right and left eigenvectors associated with the eigenvalue $\lambda_{k}$ and, throughout this section, we assume the normalization

$$
\ell_{j}^{T} \cdot \mathbf{r}_{k}=\delta_{j k}, \quad 1 \leq j, k \leq p .
$$


Lemma 1. We have for any $\mathbf{b} \in \mathbb{R}^{p}$

$$
\mathcal{O}_{R}(\mathbf{b})=\left\{\mathbf{b}+\sum_{k=1}^{q} \alpha_{k} \mathbf{r}_{k} ; \quad \alpha_{k} \in \mathbb{R}, 1 \leq k \leq q\right\}
$$

Hence the boundary condition of (3.2) at $x=0$ reads

$$
\mathbf{u}(0+, t)-\mathbf{b}(t) \in\left[\mathbf{r}_{1}, \ldots, \mathbf{r}_{q}\right]
$$

or equivalently

$$
\ell_{j}^{T} \cdot \mathbf{u}(0+, t)=\ell_{j}^{T} \cdot \mathbf{b}(t), q+1 \leq j \leq p .
$$

Then one can solve trivially problem (3.2).

Lemma 2. The solution $\mathbf{u}$ of (3.2) is given by

$$
\mathbf{u}(x, t)=\sum_{k=1}^{p} \alpha_{k}(x, t) \mathbf{r}_{k}, \quad \alpha_{k}(x, t)=\ell_{k}^{T} \cdot \mathbf{u}(x, t),
$$

where

$$
\alpha_{k}(x, t)=\left\{\begin{array}{l}
\ell_{k}^{T} \cdot \mathbf{u}_{0}\left(x-\lambda_{k} t\right), 1 \leq k \leq q, \\
\left\{\begin{array}{l}
\ell_{k}^{T} \cdot \mathbf{b}\left(t-x / \lambda_{k}\right), x<\lambda_{k} t, \\
\ell_{k}^{T} \cdot \mathbf{u}_{0}\left(x-\lambda_{k} t\right), x>\lambda_{k} t
\end{array} \quad q+1 \leq k \leq p .\right.
\end{array}\right.
$$

We obtain in particular

$$
\ell_{k}^{T} \cdot \mathbf{u}(0+, t)=\left\{\begin{array}{l}
\ell_{k}^{T} \cdot \mathbf{u}_{0}\left(-\lambda_{k} t\right), 1 \leq k \leq q, \\
\ell_{k}^{T} \cdot \mathbf{b}(t), \quad q+1 \leq k \leq p .
\end{array}\right.
$$

If $\lambda_{q}=0, \ell_{q}^{T} \cdot \mathbf{u}_{0}\left(-\lambda_{q} t\right)=\ell_{q}^{T} \cdot \mathbf{u}_{0}(0)$ should be replaced by $\ell_{k}^{T} \cdot \mathbf{u}_{0}(0+)$. We will omit this kind of correction in the sequel.

Consider on the other hand the initial boundary value problem

$$
\left\{\begin{array}{l}
\frac{\partial \mathbf{u}}{\partial t}+\mathbf{A} \frac{\partial \mathbf{u}}{\partial x}=\mathbf{0}, \quad x<0, t>0 \\
\mathbf{u}(x, 0)=\mathbf{u}_{0}(x), \quad x<0 \\
\mathbf{u}(0-, t) \in \mathcal{O}_{R}(\mathbf{b}(t)), \quad t>0
\end{array}\right.
$$

where

$$
\mathcal{O}_{L}(\mathbf{b})=\left\{\mathbf{w}=\mathbf{W}(0-; \mathbf{v}, \mathbf{b}) ; \mathbf{v} \in \mathbb{R}^{p}\right\}
$$

Here, we suppose that the eigenvalues $\lambda_{k}$ of $\mathbf{A}$ are ordered as

$$
\lambda_{1}<\lambda_{2}<\cdots<\lambda_{q}<0 \leq \lambda_{q+1}<\cdots<\lambda_{p}
$$

Then we have

$$
\ell_{k}^{T} \cdot \mathbf{u}(0-, t)=\left\{\begin{array}{l}
\ell_{k}^{T} \cdot \mathbf{b}(t), 1 \leq k \leq q, \\
\ell_{k}^{T} \cdot \mathbf{u}_{0}\left(-\lambda_{k} t\right), q+1 \leq k \leq p .
\end{array}\right.
$$

Now we pass to the coupled problem $(2.1)-(2.3),(2.6)$ where the flux functions are given by (3.1). We suppose that the eigenvalues $\lambda_{L, k}$ of the matrix $\mathbf{A}_{L}$ verify

$$
\lambda_{L, 1}<\lambda_{L, 2}<\cdots<\lambda_{L, q_{L}}<0 \leq \lambda_{L, q_{L}+1}<\cdots<\lambda_{L, p}
$$


while the eigenvalues $\lambda_{R, k}$ of the matrix $\mathbf{A}_{R}$ verify

$$
\lambda_{R, 1}<\lambda_{R, 2}<\cdots<\lambda_{R, q_{R}} \leq 0<\lambda_{R, q_{R}+1}<\cdots<\lambda_{R, p}
$$

Then we obtain from (3.4) and (3.6) that any solution of the coupled problem satisfies

$$
\ell_{L, k}^{T} \cdot \mathbf{u}(0-, t)=\left\{\begin{array}{l}
\ell_{L, k}^{T} \cdot \mathbf{u}(0+, t), 1 \leq k \leq q_{L}, \\
\ell_{L, k}^{T} \cdot \mathbf{u}_{0}\left(-\lambda_{L, k} t\right), q_{L}+1 \leq k \leq p
\end{array}\right.
$$

and

$$
\ell_{R, k}^{T} \cdot \mathbf{u}(0+, t)=\left\{\begin{array}{l}
\ell_{R, k}^{T} \cdot \mathbf{u}_{0}\left(-\lambda_{R, k} t\right), 1 \leq k \leq q_{R}, \\
\ell_{R, k}^{T} \cdot \mathbf{u}(0-, t), q_{R}+1 \leq k \leq p .
\end{array}\right.
$$

This gives a system of $2 p$ linear equations in the $2 p$ unknown components of $\mathbf{u}(0 \pm, t)$. Conversely, with any solution $\mathbf{u}(0 \pm, t)$ of $(3.9),(3.10)$, one can associate in a unique way a solution $\mathbf{u}$ of the coupled problem. Indeed the boundary values

$$
\ell_{L, k}^{T} \cdot \mathbf{u}(0-, t), 1 \leq k \leq q_{L}, \ell_{R, k}^{T} \cdot \mathbf{u}(0+, t), q_{R}+1 \leq k \leq p,
$$

together with the initial data $\mathbf{u}_{0}$ allow us to solve separately the initial boundary value problems for $x<0$ and $x>0$ and therefore the coupled problem. Thus, we have proved

Lemma 3. In the linear case (3.1), the coupled problem (2.1)-(2.3), (2.6) has a unique solution if and only if the system (3.9), (3.10) has a unique solution.

Observe that the jump

$$
\mathbf{v}(t)=\mathbf{u}(0+, t)-\mathbf{u}(0-, t)
$$

is solution of the $p+q_{L}-q_{R}$ homogeneous equations

$$
\left\{\begin{array}{l}
\ell_{L, k}^{T} \cdot \mathbf{v}(t)=0,1 \leq k \leq q_{L}, \\
\ell_{R, k}^{T} \cdot \mathbf{v}(t)=0, q_{R}+1 \leq k \leq p .
\end{array}\right.
$$

We begin by considering the simplest case $q_{L}=q_{R}$.

Theorem 1. Assume

$$
q_{L}=q_{R}=q
$$

In the linear case (3.1), the coupled problem (2.1)-(2.3), (2.6) has a unique solution if and only if $\left\{\ell_{L, 1}, \ldots \ell_{L, q}\right.$, $\left.\ell_{R, q+1}, \ldots \ell_{R, p}\right\}$ and $\left\{\ell_{R, 1}, \ldots \ell_{R, q}, \ell_{L, q+1}, \ldots \ell_{L, p}\right\}$ are two bases of $\mathbb{R}^{p}$. The coupling conditions (3.12) then read

$$
\mathbf{u}(0+, t)=\mathbf{u}(0-, t)
$$

Proof. If $\left\{\ell_{L, 1}, \ldots \ell_{L, q}, \ell_{R, q+1}, \ldots \ell_{R, p}\right\}$ is a basis of $\mathbb{R}^{p},(3.12)$ and (3.14) are equivalent. If, in addition, $\left\{\ell_{R, 1}, \ldots \ell_{R, q}, \ell_{L, q+1}, \ldots \ell_{L, p}\right\}$ is a basis of $\mathbb{R}^{p}$, the equations

$$
\left\{\begin{array}{l}
\ell_{R, k}^{T} \cdot \mathbf{u}(0, t)=\ell_{R, k}^{T} \cdot \mathbf{u}_{0}\left(-\lambda_{R, k} t\right), 1 \leq k \leq q, \\
\ell_{L, k}^{T} \cdot \mathbf{u}(0, t)=\ell_{L, k}^{T} \cdot \mathbf{u}_{0}\left(-\lambda_{L, k} t\right), q+1 \leq k \leq p,
\end{array}\right.
$$

determine $\mathbf{u}(0, t)$ in a unique way. Conversely, if the vectors $\ell_{L, 1}, \ldots \ell_{L, q}, \ell_{R, q+1}, \ldots \ell_{R, p}$ are linearly dependent, system (3.12) has nontrivial solutions and and equations. (3.9), (3.10) have not a unique solution. Similarly, if $\ell_{R, 1}, \ldots \ell_{R, q}, \ell_{L, q+1}, \ldots \ell_{L, p}$ are linearly dependent vectors, (3.15) is not a well-posed system. 
We next pass to the general case $q_{L} \neq q_{R}$. Choosing $\mathbf{v}(t)$ and $\mathbf{u}(0-, t)$ as unknowns, equations (3.9),(3.10) are equivalent to (3.12) and

$$
\left\{\begin{array}{l}
\ell_{L, k}^{T} \cdot \mathbf{u}(0-, t)=\ell_{L, k}^{T} \cdot \mathbf{u}_{0}\left(-\lambda_{L, k} t\right), q_{L}+1 \leq k \leq p \\
\ell_{R, k}^{T} \cdot \mathbf{u}(0-, t)+\ell_{R, k}^{T} \cdot \mathbf{v}(t)=\ell_{R, k}^{T} \cdot \mathbf{u}_{0}\left(-\lambda_{R, k} t\right), 1 \leq k \leq q_{R} .
\end{array}\right.
$$

We first study the case $q_{R}>q_{L}$. We set

$$
q=q_{R}=q_{L}+m, m \geq 1
$$

Lemma 4. Assume (3.17). Then a necessary condition for the system (3.9), (3.10) to have a unique solution is that the $p-m$ vectors $\ell_{L, 1}, \ldots \ell_{L, q-m}, \ell_{R, q+1}, \ldots \ell_{R, p}$ are linearly independent.

Proof. With condition (3.17), we have $\ell_{L, q-m}=\ell_{L, q_{L}}$ and $\ell_{R, q+1}=\ell_{R, q_{R}+1}$. If the vectors $\ell_{L, 1}, \ldots, \ell_{L, q_{L}}$, $\ell_{R, q_{R}+1}, \ldots \ell_{R, p}$ are linearly dependent, the $2 p \times 2 p$ linear system (3.12), (3.16) is clearly undetermined, which proves our assertion.

Let us introduce the space

We observe that on the one hand

$$
E=\left[\mathbf{r}_{R, 1}, \ldots \mathbf{r}_{R, q}\right] \cap\left[\mathbf{r}_{L, q-m+1}, \ldots, \mathbf{r}_{L, p}\right] .
$$

$$
\mathbf{v}(t) \in E \Longleftrightarrow \mathbf{v}(t) \text { satisfies equations (3.12), }
$$

and on the other hand the vectors $\ell_{L, 1}, \ldots \ell_{L, q-m}, \ell_{R, q+1}, \ldots \ell_{R, p}$ are linearly independent if and only

$$
\operatorname{dim} E=m \text {. }
$$

Assuming the condition (3.19), it remains to give simple algebraic criteria which ensure that the system (3.9), (3.10) (or (3.12), (3.16)) has a unique solution.

Let us first examine two trivial situations.

Example 3.1. $q_{R}=p$. We have in this case

$$
E=\left[\mathbf{r}_{L, p-m+1}, \ldots, \mathbf{r}_{L, p}\right], \quad \operatorname{dim} E=m,
$$

and the coupled problem has a unique solution (3.9). Indeed (3.10) gives

$$
\mathbf{u}(0+, t)=\sum_{k=1}^{p} \ell_{R, k}^{T} \cdot \mathbf{u}_{0}\left(-\lambda_{R, k} t\right) \mathbf{r}_{R, k},
$$

and (3.9) determines $\mathbf{u}(0-, t)$.

Example 3.2. $q_{L}=0$. This is the symmetric case of that of the previous example. We have here

$$
E=\left[\mathbf{r}_{R, 1}, \ldots, \mathbf{r}_{R, m}\right], \quad \operatorname{dim} E=m .
$$

Again the coupled problem has a unique solution, (3.9) determines $\mathbf{u}(0-, t)$ and then (3.10) gives $\mathbf{u}(0+, t)$.

Example 3.1 (resp. Ex. 3.2) corresponds to the case where the problem

$$
\left\{\begin{array}{l}
\frac{\partial \mathbf{u}}{\partial t}+\mathbf{A}_{R} \frac{\partial \mathbf{u}}{\partial x}=\mathbf{0}, \quad x>0, t>0 \\
\mathbf{u}(x, 0)=\mathbf{u}_{0}(x), x>0
\end{array}\right.
$$


(resp. the problem

$$
\left\{\begin{array}{l}
\frac{\partial \mathbf{u}}{\partial t}+\mathbf{A}_{L} \frac{\partial \mathbf{u}}{\partial x}=\mathbf{0}, \quad x<0, t>0, \\
\mathbf{u}(x, 0)=\mathbf{u}_{0}(x), x<0
\end{array}\right.
$$

is well posed since all the characteristics are outgoing at the boundary. Coupling the two problems is then obvious!

Let us go back to the general situation (3.17) with the assumption (3.19). In addition, we make the following assumption

$$
\text { the } p \text { vectors } \ell_{R, 1}, \ldots \ell_{R, q-m}, \ell_{L, q-m+1}, \ldots \ell_{L, p} \text { are linearly independent. }
$$

Then we may write

$$
\ell_{L, k}=\sum_{j=1}^{q-m} \beta_{j, k} \ell_{R, j}+\sum_{j=q-m+1}^{p} \beta_{j, k} \ell_{L, j}, 1 \leq k \leq q-m .
$$

Lemma 5. Assume (3.20). Then the $(q-m) \times(q-m)$ matrix $\left(\beta_{i, j}\right)_{1 \leq i, j \leq q-m}$ is invertible and its inverse matrix $\left(\alpha_{i, j}\right)_{1 \leq i, j \leq q-m}$ is given by

$$
\alpha_{i, j}=\ell_{R, j}^{T} \cdot \mathbf{r}_{L, i}
$$

Proof. Using (3.21), we have

$$
\ell_{R, j}^{T} \cdot \mathbf{r}_{L, i}=\sum_{j=1}^{q-m} \beta_{j, k} \ell_{R, j}^{T} \cdot \mathbf{r}_{L, i}+\sum_{j=q-m+1}^{p} \beta_{j, k} \ell_{L, j}^{T} \cdot \mathbf{r}_{L, i},
$$

and by (3.3)

which proves the result.

$$
\delta_{i, k}=\sum_{j=1}^{q-m} \beta_{j, k} \ell_{R, j}^{T} \cdot \mathbf{r}_{L, i}
$$

We next introduce the subspace

$$
F=\left\{\ell \in\left[\ell_{R, 1}, \ldots, \ell_{R, q}\right] ; \ell^{T} \cdot \mathbf{r}_{L, i}=0,1 \leq i \leq q-m\right\}
$$

Lemma 6. Assume the hypothesis (3.20). Then $\operatorname{dim} F=m$ and $F$ is spanned by the vectors

$$
\ell_{i}=\ell_{R, i}-\sum_{j, k=1}^{q-m} \beta_{j, k}\left(\ell_{R, i}^{T} \cdot \mathbf{r}_{L, k}\right) \ell_{R, j}, \quad q-m+1 \leq i \leq q .
$$

Proof. Let

$$
\ell=\sum_{j=1}^{q} \gamma_{j} \ell_{R, j} \in F
$$

we have

$$
\sum_{j=1}^{q-m} \gamma_{j} \ell_{R, j}^{T} \cdot \mathbf{r}_{L, i}=-\sum_{j=q-m+1}^{q} \gamma_{j} \ell_{R, j}^{T} \cdot \mathbf{r}_{L, i}, \quad 1 \leq i \leq q-m
$$

and by Lemma 5 ,

$$
\gamma_{j}=-\sum_{k=1}^{q-m} \beta_{j, k} \sum_{s=q-m+1}^{q} \gamma_{s} \ell_{R, s}^{T} \cdot \mathbf{r}_{L, k}, \quad 1 \leq j \leq q-m .
$$

The result follows by choosing $\gamma_{s}=\delta_{i, s}, q-m+1 \leq i, s \leq q$. 
Let $E^{\circ}$ denote the orthogonal of $E$ in $\mathbb{R}^{p}$. We are now able to state

Theorem 2. Assume the hypotheses (3.17) and (3.19) together with (3.1). Then under the condition (3.20), the coupled problem (2.1)-(2.3), (2.6) has a unique solution if and only if the subspaces $E$ and $F$ satisfy $E^{\circ} \cap F=\{0\}$.

Proof. Let us derive an equation for $\mathbf{v}(t) \in E$. Using (3.16) for $q_{L}+1=q-m+1 \leq k \leq p$, we write

$$
\begin{aligned}
\mathbf{u}(0-, t) & =\sum_{k=1}^{p} \ell_{L, k}^{T} \cdot \mathbf{u}(0-, t) \mathbf{r}_{L, k} \\
& =\sum_{k=1}^{q-m} \ell_{L, k}^{T} \cdot \mathbf{u}(0-, t) \mathbf{r}_{L, k}+\sum_{k=q-m+1}^{p} \ell_{L, k}^{T} \cdot \mathbf{u}_{0}\left(-\lambda_{L, k} t\right) \mathbf{r}_{L, k}
\end{aligned}
$$

On the other hand, using (3.21) we have

$$
\sum_{k=1}^{q-m} \ell_{L, k}^{T} \cdot \mathbf{u}(0-, t) \mathbf{r}_{L, k}=\sum_{j, k=1}^{q-m} \beta_{j, k} \ell_{R, j}^{T} \cdot \mathbf{u}(0-, t) \mathbf{r}_{L, k}+\sum_{k=1}^{q-m} \sum_{j=q-m+1}^{p} \beta_{j, k} \ell_{L, j}^{T} \cdot \mathbf{u}(0-, t) \mathbf{r}_{L, k}
$$

and by using again (3.16)

$$
\begin{aligned}
\sum_{k=1}^{q-m} \ell_{L, k}^{T} \cdot \mathbf{u}(0-, t) \mathbf{r}_{L, k}= & \sum_{j, k=1}^{q-m} \beta_{j, k} \ell_{R, j}^{T} \cdot \mathbf{u}_{0}\left(-\lambda_{R, j} t\right) \mathbf{r}_{L, k} \\
& -\sum_{j, k=1}^{q-m} \beta_{j, k} \ell_{R, j}^{T} \cdot \mathbf{v}(t) \mathbf{r}_{L, k}+\sum_{k=1}^{q-m} \sum_{j=q-m+1}^{p} \beta_{j, k} \ell_{L, j}^{T} \cdot \mathbf{u}_{0}\left(-\lambda_{L, j} t\right) \mathbf{r}_{L, k} .
\end{aligned}
$$

Thus we obtain

$$
\begin{aligned}
\mathbf{u}(0-, t)= & -\sum_{j, k=1}^{q-m} \beta_{j, k} \ell_{R, j}^{T} \cdot \mathbf{v}(t) \mathbf{r}_{L, k}+\sum_{k=1}^{q-m}\left\{\sum_{j=1}^{q-m} \beta_{j, k} \ell_{R, j}^{T} \cdot \mathbf{u}_{0}\left(-\lambda_{R, j} t\right)+\sum_{j=q-m+1}^{p} \beta_{j, k} \ell_{L, j}^{T} \cdot \mathbf{u}_{0}\left(-\lambda_{L, j} t\right)\right\} \mathbf{r}_{L, k} \\
& +\sum_{j=q-m+1}^{p} \ell_{L, k}^{T} \cdot \mathbf{u}_{0}\left(-\lambda_{L, k} t\right) \mathbf{r}_{L, k}
\end{aligned}
$$

At that stage, we remark that we have not yet used in (3.16) the equations corresponding to $q_{L}+1=q-m+1 \leq k \leq q=q_{R}$. By replacing $\mathbf{u}(0-, t)$ by the above expression in these equations, we find

$$
\begin{aligned}
\ell_{R, i}^{T} \cdot \mathbf{v}(t)-\sum_{j, k=1}^{q-m} \beta_{j, k}\left(\ell_{R, i}^{T} \cdot \mathbf{r}_{L, k}\right) \ell_{R, j}^{T} \cdot \mathbf{v}(t)= & -\sum_{k=1}^{q-m}\left\{\sum_{j=1}^{q-m} \beta_{j, k} \ell_{R, j}^{T} \cdot \mathbf{u}_{0}\left(-\lambda_{R, k} t\right)\right. \\
& \left.+\sum_{j=q-m+1}^{p} \beta_{j, k} \ell_{L, j}^{T} \cdot \mathbf{u}_{0}\left(-\lambda_{L, j} t\right)\right\} \ell_{R, i}^{T} \mathbf{r}_{L, k} \\
& +\ell_{R, i}^{T} \cdot \mathbf{u}_{0}\left(-\lambda_{R, i} t\right) \\
& -\sum_{k=q-m+1}^{p} \ell_{L, k}^{T} \cdot \mathbf{u}_{0}\left(-\lambda_{L, k} t\right) \ell_{R, i}^{T} \cdot \mathbf{r}_{L, k}, \quad q-m+1 \leq i \leq q .
\end{aligned}
$$


In other words, we obtain

$$
\ell_{i}^{T} \cdot \mathbf{v}(t)=\text { known right-hand side, } q-m+1 \leq i \leq q
$$

where the $\ell_{i}$ 's are defined as in (3.23). It follows from Lemma 6 that this system of $m$ equations has a unique solution $\mathbf{v}(t) \in E$ if and only if $E$ and $F$ satisfy the condition of nonorthogonality $E^{\circ} \cap F=\{0\}$ where $E^{\circ}$ denotes the orthogonal of $E$ in $\mathbb{R}^{p}$. Once $\mathbf{v}(t)$ is known, we get $\mathbf{u}(0, t)$ trivially.

A simple sufficient condition is $\mathbf{r}_{R, i}=\mathbf{r}_{L, i}, i=q-m+1, \ldots q$ and $\ell_{R, i}=\ell_{L, i}, i=q-m+1, \ldots q$ since $E=\left[\mathbf{r}_{R, q-m+1}, \ldots \mathbf{r}_{R, q}\right]=\left[\mathbf{r}_{L, q-m+1}, \ldots \mathbf{r}_{L, q}\right], F=\left[\ell_{R, q-m+1}, \ldots \ell_{R, q}\right]=\left[\ell_{L, q-m+1}, \ldots \ell_{L, q}\right]$, and $E^{\circ}=\left[\ell_{R, 1}, \ldots \ell_{R, q-m}, \ell_{R, q+1}, \ldots \ell_{R, p}\right]=\left[\ell_{L, 1}, \ldots \ell_{L, q-m}, \ell_{L, q+1}, \ldots \ell_{L, p}\right]$ so that $E^{\circ} \cap F=\{0\}$.

We will see in next section an illustration of a more complex situation with $q=q_{R}=q_{L}+1=2$, $\operatorname{dim} E=m=1$ but $\mathbf{r}_{R, 2}$ and $\mathbf{r}_{L, 2}$ do not coincide.

It remains to consider the case

$$
q_{L}>q_{R}
$$

Then the solution of the coupled problem is not unique in general. Let us make the following natural assumptions

$$
\begin{aligned}
& \text { the } p+q_{L}-q_{R} \text { vectors } \ell_{L, 1}, \ldots, \ell_{L, q_{L}}, \ell_{R, q_{R}+1}, \ldots, \ell_{R, p} \text { span } \mathbb{R}^{p}, \\
& \left\{\begin{array}{l}
\text { the } p-\left(q_{L}-q_{R}\right) \text { vectors } \ell_{R, 1}, \ldots, \ell_{R, q_{R}}, \ell_{L, q_{L}+1}, \ldots, \ell_{L, p} \text { are } \\
\text { linearly independent. }
\end{array}\right.
\end{aligned}
$$

We choose $q_{L}-q_{R}$ vectors $\ell_{q_{R}+1}, \ldots, \ell_{q_{L}}$ in such a way that the $p$ vectors $\ell_{R, 1}, \ldots, \ell_{R, q_{R}}, \ell_{q_{R}+1}, \ldots, \ell_{q_{L}}, \ell_{L, q_{L}+1}$, $\ldots, \ell_{L, p}$ form a basis of $\mathbb{R}^{p}$.

Theorem 3. Assume (3.1), (3.24) and the hypotheses (3.25) and (3.26). Then the solutions of the coupled problem (2.1)-(2.3), (2.6) form an affine variety of dimension $q_{L}-q_{R}$. Any solution $\mathbf{u}$ satisfies (3.14). Moreover such a solution is uniquely determined if, in addition, we prescribe the values of

$$
\ell_{k}^{T} \cdot \mathbf{u}(0, t), \quad q_{R}+1 \leq k \leq q_{L},
$$

at the interface $x=0$.

Proof. Under the hypothesis (3.25), equations (3.12) yield

$$
\mathbf{v}(t)=\mathbf{0},
$$

so that (3.14) holds and (3.16) becomes

$$
\left\{\begin{array}{l}
\ell_{L, k}^{T} \cdot \mathbf{u}(0, t)=\ell_{L, k}^{T} \cdot \mathbf{u}_{0}\left(-\lambda_{L, k} t\right), \quad q_{L}+1 \leq k \leq p \\
\ell_{R, k}^{T} \cdot \mathbf{u}(0, t)+\ell_{R, k}^{T} \cdot \mathbf{v}(t)=\ell_{R, k}^{T} \cdot \mathbf{u}_{0}\left(-\lambda_{R, k} t\right), \quad 1 \leq k \leq q_{R}
\end{array}\right.
$$

Together with prescribed values of $\ell_{k}^{T} \cdot \mathbf{u}(0, t), q_{R}+1 \leq k \leq q_{L}$, this determines $\mathbf{u}(0, t)$ in a unique way as soon as (3.26) holds. 


\section{Application to THE LinEARIZED SYSTEM OF GAS DYNAMiCS IN LAGRANGiAN COORDINATES}

Consider the system of gas dynamics in Lagrangian coordinates

$$
\frac{\partial \mathbf{u}}{\partial t}+\frac{\partial}{\partial x} \mathbf{f}(\mathbf{u})=\mathbf{0}
$$

where

$$
\mathbf{u}=(\tau, v, e)^{T}, \mathbf{f}(\mathbf{u})=(-v, p, p v)^{T}
$$

In (4.1), $x$ stands for a mass variable while in (4.2), $\tau$ denotes the specific volume, $v$ the velocity, $e=\varepsilon+\frac{1}{2} v^{2}$ the specific total energy, $\varepsilon$ the specific internal energy, and $p=p(\tau, \varepsilon)$. In Sections 4 and 5 , we study the coupling of two such systems at a contact discontinuity located at $x=0$ and separating two fluids with different equations of state $p=p_{\alpha}(\tau, \varepsilon), \alpha=L, R$. We denote by $\mathbf{f}_{\alpha}(\mathbf{u}), \alpha=L, R$, the corresponding flux functions.

Let $\mathbf{u}_{L}$ and $\mathbf{u}_{R}$ be two constant states separated by such a contact discontinuity, i.e., we have continuity of the 2-Riemann invariants

$$
\left\{\begin{array}{l}
v_{L}=v_{R} \\
p_{L}\left(\tau_{L}, \varepsilon_{L}\right)=p_{R}\left(\tau_{R}, \varepsilon_{R}\right)
\end{array}\right.
$$

In this section, we consider the coupling problem for the gas dynamics system linearized at the states $\mathbf{u}_{L}$ and $\mathbf{u}_{R}$ respectively and we show that it is indeed well-posed. The situation is that of Section 3 with

$$
\mathbf{A}_{\alpha}=\mathbf{A}_{\alpha}\left(\mathbf{u}_{\alpha}\right), \alpha=L, R
$$

where $\mathbf{A}(\mathbf{u})$ is the Jacobian matrix of $\mathbf{f}(\mathbf{u})$

$$
\left(\begin{array}{ccc}
0 & -1 & 0 \\
p_{\tau} & -v p_{\varepsilon} & p_{\varepsilon} \\
v p_{\tau} & p-v^{2} p_{\varepsilon} & v p_{\varepsilon}
\end{array}\right)
$$

with the notations

Here $p=3$ and

$$
p_{\varepsilon}=\frac{\partial p}{\partial \varepsilon}(\tau, \varepsilon), p_{\tau}=\frac{\partial p}{\partial \tau}(\tau, \varepsilon)
$$

$$
\lambda_{1}=-C<\lambda_{2}=0<\lambda_{3}=C,
$$

where

$$
C=\sqrt{-p_{\tau}+p p_{\varepsilon}}
$$

denotes the Lagrangian sound speed. Recall that the right eigenvectors of $\mathbf{A}(\mathbf{u})$ can be chosen as

$$
\mathbf{r}_{1}=\left(\begin{array}{c}
-1 \\
-C \\
p-C v
\end{array}\right), \quad \mathbf{r}_{2}=\left(\begin{array}{c}
p_{\varepsilon} \\
0 \\
-p_{\tau}
\end{array}\right), \quad \mathbf{r}_{3}=\left(\begin{array}{c}
-1 \\
C \\
p+C v
\end{array}\right)
$$

while the left eigenvectors are given by

$$
\ell_{1}=\frac{1}{2 C^{2}}\left(\begin{array}{c}
p_{\tau} \\
-C-v p_{\varepsilon} \\
p_{\varepsilon}
\end{array}\right), \quad \ell_{2}=\frac{1}{C^{2}}\left(\begin{array}{c}
p \\
-v \\
1
\end{array}\right), \quad \ell_{3}=\frac{1}{2 C^{2}}\left(\begin{array}{c}
p_{\tau} \\
C-v p_{\varepsilon} \\
p_{\varepsilon}
\end{array}\right) .
$$

In this case, we have $q_{L}=1$ and $q_{R}=q=2$. We are therefore in the situation (3.17) with $m=1$. Let us check that the dimension of the subspace

$$
E=\left[\mathbf{r}_{R, 1}, \mathbf{r}_{R, 2}\right] \cap\left[\mathbf{r}_{L, 2}, \mathbf{r}_{L, 3}\right]
$$


is equal to 1 . Let $\mathbf{r} \in E$; we write

$$
\mathbf{r}=\beta_{1} \mathbf{r}_{R, 1}+\beta_{2} \mathbf{r}_{R, 2}=\gamma_{2} \mathbf{r}_{L, 2}+\gamma_{3} \mathbf{r}_{L, 3}
$$

The quadruple $\left(\beta_{1}, \beta_{2}, \gamma_{1}, \gamma_{2}\right)$ is solution of the system of equations

$$
\left\{\begin{array}{l}
-\beta_{1}+\beta_{2}\left(p_{\varepsilon}\right)_{R}=\gamma_{2}\left(p_{\varepsilon}\right)_{L}-\gamma_{3} \\
-C_{R} \beta_{1}=C_{L} \gamma_{3} \\
\left(p-C_{R} v\right) \beta_{1}-\beta_{2}\left(p_{\tau}\right)_{R}=-\gamma_{2}\left(p_{\tau}\right)_{L}+\left(p+C_{L} v\right) \gamma_{3}
\end{array}\right.
$$

Multiplying the first equation by $p$ and adding it to the third equation, we obtain

$$
-C_{R} v \beta_{1}+C_{R}^{2} \beta_{2}=C_{L}^{2} \gamma_{2}+C_{L} v \gamma_{3}
$$

and by the second equation

$$
C_{R}^{2} \beta_{2}=C_{L}^{2} \gamma_{2}
$$

Then, choosing $\beta_{2}$ as a parameter, we find that the pair $\left(\beta_{1}, \gamma_{3}\right)$ is the unique solution of the system

$$
\left\{\begin{array}{l}
\beta_{1}-\gamma_{3}=\left(\left(p_{\varepsilon}\right)_{R}-\frac{C_{R}^{2}}{C_{L}^{2}}\left(p_{\varepsilon}\right)_{L}\right) \beta_{2} \\
C_{R} \beta_{1}+C_{L} \gamma_{3}=0
\end{array}\right.
$$

which proves that $\operatorname{dim} E=1$.

Next, a simple calculation gives

$$
\operatorname{det}\left(\ell_{R, 1}, \ell_{L, 2}, \ell_{L, 3}\right)=\frac{1}{4 C_{R} C_{L}^{3}}\left(C_{R}+C_{L}\right)>0,
$$

so that assumption (3.20) holds. Then, by Lemma 6 , the subspace

$$
F=\left\{\ell \in\left[\ell_{R, 1}, \ell_{R, 2}\right] ; \ell^{T} \cdot \mathbf{r}_{L, 1}=0\right\}
$$

is one-dimensional. Since

$$
\ell_{R, 2}^{T} \cdot \mathbf{r}_{L, 1}=0
$$

we find that $F$ is spanned by $\ell_{R, 2}$. Moreover, if $\mathbf{r}$ belongs to $E$, i.e., is of the form (4.5), we have

$$
\ell_{R, 2}^{T} \cdot \mathbf{r}=\beta_{2},
$$

which proves that $E$ and $F$ are not orthogonal. The well-posedness of the corresponding coupled problem then follows from Theorem 2. We have thus proved that imposing the continuity of the pressure and velocity at the material interface leads to two well-posed linearized boundary value problems.

Remark 5. Note that $\ell_{L, 2}$ is parallel to $\ell_{R, 2}$ which implies that

$$
\left[\mathbf{r}_{L, 1}, \mathbf{r}_{L, 3}\right]=\left[\mathbf{r}_{R, 1}, \mathbf{r}_{R, 3}\right]
$$

but $\mathbf{r}_{L, 2}$ and $\mathbf{r}_{R, 2}$ do not coincide.

For instance for two ideal gases $p=(\gamma-1) \varepsilon / \tau$ with $\gamma=\gamma_{L}$ or $\gamma_{R}$, so that $\frac{\partial p}{\partial \tau}=-p / \tau, \frac{\partial p}{\partial \varepsilon}=p / \varepsilon$, we get

$$
\mathbf{r}_{2}=\frac{p}{\varepsilon}\left(\begin{array}{c}
1 \\
0 \\
-p /(\gamma-1)
\end{array}\right)
$$


which obviously depends on $\gamma$. However the intersection $E$ of the planes $\left[\mathbf{r}_{R, 1}, \mathbf{r}_{R, 2}\right]$ and $\left[\mathbf{r}_{L, 2}, \mathbf{r}_{L, 3}\right]$ is indeed a line, since if it were a plane, it would contain $\mathbf{r}_{R, 1}, \mathbf{r}_{R, 2}, \mathbf{r}_{L, 2}, \mathbf{r}_{L, 3}$ and thus the whole space.

\section{The COUPLing OF FLUID MODEls AT A MATERIAL CONTACT DISCONTINUITY}

We pass to the coupling of nonlinear hyperbolic systems. We consider in this section the coupling of two gas dynamics systems at a contact discontinuity. Again, we use Lagrangian coordinates so that the contact discontinuity is fixed and located at $x=0$. We begin by considering the problem (2.1)-(2.3), (2.6) where

$$
\mathbf{u}=(\tau, v, e)^{T}, \mathbf{f}_{\alpha}(\mathbf{u})=(-v, p, p v)^{T}, p=p_{\alpha}(\tau, \varepsilon), \alpha=L, R .
$$

Given a left state $\mathbf{u}_{L}$, we denote by $\mathcal{S}_{R}^{1}\left(\mathbf{u}_{L}\right)$ the 1 -wave curve consisting of states $\mathbf{u}$ which can be connected to $\mathbf{u}_{L}$ on the right by either a 1 -shock or a 1 -rarefaction wave corresponding to the equation of state $p=p_{R}(\tau, \varepsilon)$. Similarly, given a right state $\mathbf{u}_{R}$, we denote by $\mathcal{S}_{L}^{3}\left(\mathbf{u}_{R}\right)$, the 3 -wave curve consisting of states $\mathbf{u}$ which can be connected to $\mathbf{u}_{R}$ on the left by a 3-shock or a 3-rarefaction wave corresponding to the equation of state $p=p_{L}(\tau, \varepsilon)$. We denote by $\mathbf{S}_{R}^{1}\left(\mathbf{u}_{L}\right)$ and $\mathbf{S}_{L}^{3}\left(\mathbf{u}_{R}\right)$ the projections onto the $(v, p)$-plane of the wave curves $\mathcal{S}_{R}^{1}\left(\mathbf{u}_{L}\right)$ and $\mathcal{S}_{L}^{3}\left(\mathbf{u}_{R}\right)$ respectively. We then make the following hypothesis which is indeed satisfied for "standard" equations of state

$$
\left\{\begin{array}{l}
\text { for any pair of states }\left(\mathbf{u}_{L}, \mathbf{u}_{R}\right), \text { the curves } \\
\mathbf{S}_{R}^{1}\left(\mathbf{u}_{L}\right) \text { and } \mathbf{S}_{L}^{3}\left(\mathbf{u}_{R}\right) \text { may intersect at one point at most. }
\end{array}\right.
$$

Lemma 7. Assume the hypothesis (5.2). Then, in the case (5.1), the coupling conditions (2.6) are equivalent to

$$
\left\{\begin{array}{l}
v(0+, t)=v(0-, t) \\
p(0+, t)=p(0-, t) .
\end{array}\right.
$$

Proof. By using the structure of the solution of the Riemann problem for the gas dynamics equations, the condition $\mathbf{u}(0-, t) \in \mathcal{O}_{L}(\mathbf{u}(0+, t))$ simply means that $(v, p)(0-, t) \in \mathbf{S}_{L}^{3}(\mathbf{u}(0+, t))$. Similarly, the condition $\mathbf{u}(0+, t) \in \mathcal{O}_{R}(\mathbf{u}(0-, t))$ means that $(v, p)(0+, t) \in \mathbf{S}_{R}^{1}(\mathbf{u}(0-, t))$. If (5.2) holds, then $(v, p)(0+, t)$ and $(v, p)(0-, t)$ must necessarily coincide which proves the lemma. Note that the coupling conditions have been expressed using the variables $(u, p)$ (we refer to [6] for a more general approach).

Remark 6. Condition (5.2) can be connected to the condition (3.19) for the linearized problem. It is convenient here to use the set of variables

$$
\tilde{\mathbf{u}}=(\tau, v, p)^{T}
$$

and the corresponding nonconservative form of the gas dynamics equations

$$
\frac{\partial \tilde{\mathbf{u}}}{\partial t}+\tilde{\mathbf{A}}(\tilde{\mathbf{u}}) \frac{\partial \tilde{\mathbf{u}}}{\partial x}=\mathbf{0}
$$

with

$$
\tilde{\mathbf{A}}(\tilde{\mathbf{u}})=\left(\begin{array}{ccc}
0 & -1 & 0 \\
0 & 0 & 1 \\
0 & C^{2} & 0
\end{array}\right) .
$$

The (right) eigenvectors $\tilde{\mathbf{r}}_{j}=\tilde{\mathbf{r}}_{j}(\tilde{\mathbf{u}})$ of the matrix $\tilde{\mathbf{A}}=\tilde{\mathbf{A}}(\tilde{\mathbf{u}})$ may be chosen as

$$
\tilde{\mathbf{r}}_{1}=\left(\begin{array}{c}
-1 \\
-C \\
C^{2}
\end{array}\right), \quad \tilde{\mathbf{r}}_{2}=\left(\begin{array}{l}
1 \\
0 \\
0
\end{array}\right), \quad \tilde{\mathbf{r}}_{3}=\left(\begin{array}{c}
-1 \\
C \\
C^{2}
\end{array}\right) .
$$


Again, we consider two states $\mathbf{u}_{L}$ and $\mathbf{u}_{R}$ separated by a contact discontinuity so that (4.3) holds. We set

$$
\tilde{\mathbf{A}}_{\alpha}=\tilde{\mathbf{A}}_{\alpha}\left(\tilde{\mathbf{u}}_{\alpha}\right), \alpha=L, R
$$

and we denote by $\tilde{\mathbf{r}}_{\alpha, j}$ the (right) eigenvectors of $\tilde{\mathbf{A}}_{\alpha}, \alpha=L, R$, and $P \tilde{\mathbf{r}}_{\alpha, j}$ their projections onto the $(v, p)$-plane. Then, at the point $\left(v_{L}, p_{L}\right)=\left(v_{R}, p_{R}\right)$, the curves $\mathbf{S}_{R}^{1}\left(\mathbf{u}_{L}\right)$ and $\mathbf{S}_{L}^{3}\left(\mathbf{u}_{R}\right)$ are tangent to the vectors $P \tilde{\mathbf{r}}_{R, 1}$ and $P \tilde{\mathbf{r}}_{L, 3}$ respectively. Hence the linearized analog of (5.2) reads:

$$
\text { the vectors } P \tilde{\mathbf{r}}_{R, 1} \text { and } P \tilde{\mathbf{r}}_{L, 3} \text { are not colinear. }
$$

Observe that $\tilde{\mathbf{r}}_{L, 2}=\tilde{\mathbf{r}}_{R, 2}=\tilde{\mathbf{r}}_{2}$ is orthogonal to the $(v, p)$-plane so that the condition (5.5) amounts to suppose that the subspace

$$
\tilde{E}=\left[\tilde{\mathbf{r}}_{R, 1}, \tilde{\mathbf{r}}_{R, 2}\right] \cap\left[\tilde{\mathbf{r}}_{L, 2}, \tilde{\mathbf{r}}_{L, 3}\right]
$$

is spanned by the vector $\tilde{\mathbf{r}}_{2}$ or equivalently that $\operatorname{dim} \tilde{E}=1$.

Lastly, we remark that condition (5.5) is trivially satisfied.

It follows from Lemma 7 that it is equivalent to look for a solution of the coupled problem (2.1)-(2.3), (2.6) or of the system of conservation laws

$$
\left\{\begin{array}{l}
\frac{\partial \mathbf{u}}{\partial t}+\frac{\partial}{\partial x} \mathbf{f}(x, \mathbf{u})=\mathbf{0} \\
\mathbf{u}(x, 0)=\mathbf{u}_{0}(x)
\end{array}\right.
$$

where the flux function $\mathbf{f}(x, \mathbf{u})$ is defined by

$$
\mathbf{f}(x, \mathbf{u})=\left\{\begin{array}{l}
\mathbf{f}_{L}(\mathbf{u}), x<0 \\
\mathbf{f}_{R}(\mathbf{u}), x>0
\end{array}\right.
$$

Note that the conditions (5.3) imply that

$$
\mathbf{f}_{L}(\mathbf{u}(0-, t))=\mathbf{f}_{R}(\mathbf{u}(0+, t)),
$$

so that $x=0$ is a contact discontinuity between the two fluids.

Recall that the system $(2.1),(2.2)$ with (4.2) and the two corresponding equations of state $p_{L}, p_{R}$, can be put in a canonical form called the extended system of gas dynamics. Introduce a smooth function $p=p(\tau, \varepsilon, \phi)$ such that

$$
p(\tau, \varepsilon, \phi)=\left\{\begin{array}{l}
p_{L}(\tau, \varepsilon), \phi=\phi_{L} \\
p_{R}(\tau, \varepsilon), \phi=\phi_{R}
\end{array}\right.
$$

for some values $\phi_{L}$ and $\phi_{R}$ of $\phi$. The extended system of gas dynamics in Lagrangian coordinates may then be written

where

$$
\frac{\partial \mathbf{U}}{\partial t}+\frac{\partial}{\partial x} \mathbf{F}(\mathbf{U})=\mathbf{0}
$$

$$
\mathbf{U}=(\tau, v, e, \phi)^{T}, \mathbf{F}(\mathbf{U})=(-v, p, p v, 0)^{T} .
$$

The function $\phi$ is called a "colour function" and can be chosen in a number of ways (see [25] for an extensive discussion of possible color functions or [1] and the references therein). System (5.9)(5.10) is hyperbolic, with the eigenvalues $-C, 0,0, C$ and eigenvectors

$$
\mathbf{R}_{i}=\left(\begin{array}{c}
\mathbf{r}_{i} \\
0
\end{array}\right), i=1,2,3,
$$

associated to the eigenvalues $-C, 0, C$, where the $\mathbf{r}_{i}$ are given by (4.4) and a fourth eigenvector associated to 0 which may be taken as $\mathbf{R}_{2, \phi}=\left(0,0, p_{\phi},-p_{\varepsilon}\right)^{T}$. A solution of the conservative system associated to a 
discontinuous initial data across $x=0$, with $\phi=\phi_{L}, x<0$, and $\phi=\phi_{R}, x>0$, has a contact discontinuity satisfying $[\mathbf{F}(\mathbf{U})]=\mathbf{0}$ on $x=0$ so that (5.3) holds as well as (2.1), (2.2).

Hence, there are three ways of numerically solving the interface problem for the system of gas dynamics: two classical ways are based on either the direct formulation (5.6), (5.7) or the extended formulation (5.8), (5.9), (5.10); a nonconservative way is based on the coupled problem and uses the general numerical method (2.8)-(2.9) of solution of coupled problems. We refer to [1] for a discussion of the corresponding methods in Eulerian coordinates.

We can also generalize the above approach in order to couple different fluid models at a contact discontinuity. Consider the coupling of the isentropic gas dynamics equations with the general gas dynamics equations. The isentropic system in Lagrangian coordinates reads

$$
\left\{\begin{array}{l}
\frac{\partial \tau}{\partial t}-\frac{\partial v}{\partial x}=0 \\
\frac{\partial v}{\partial t}+\frac{\partial p}{\partial x}=0
\end{array}\right.
$$

where

$$
p=p\left(\tau, s_{0}\right)
$$

for some fixed value $s_{0}$ of the specific entropy and $p=p(\tau, s)$ is the equation of state of the fluid expressed in terms of $\tau$ and $s$. An extended form of the isentropic model consists in solving

$$
\left\{\begin{array}{l}
\frac{\partial \tau}{\partial t}-\frac{\partial v}{\partial x}=0 \\
\frac{\partial v}{\partial t}+\frac{\partial p}{\partial x}=0 \\
\frac{\partial s}{\partial t}=0
\end{array}\right.
$$

with $p=p(\tau, s)$. We then consider the coupled problem

$$
\left\{\begin{array}{l}
\frac{\partial \mathbf{w}}{\partial t}+\frac{\partial}{\partial x} \mathbf{f}_{L}(\mathbf{w})=\mathbf{0}, x<0 \\
\frac{\partial \mathbf{u}}{\partial t}+\frac{\partial}{\partial x} \mathbf{f}_{R}(\mathbf{u})=\mathbf{0}, x>0
\end{array}\right.
$$

where

$$
\mathbf{u}=(\tau, v, e)^{T}, \mathbf{w}=(\tau, v, s)^{T}
$$

and

$$
\mathbf{f}_{L}(\mathbf{w})=(-v, p, 0)^{T}, \mathbf{f}_{R}(\mathbf{u})=(-v, p, p v)^{T},
$$

and the systems have now the same size. As coupling conditions, we take

$$
\left\{\begin{array}{l}
\mathbf{w}(0-, t) \in \mathcal{O}_{L}(\mathbf{w}(0+, t)), \mathbf{w}(0+, t)=\mathbf{w}(\mathbf{u}(0+, t)), \\
\mathbf{u}(0+, t) \in \mathcal{O}_{R}(\mathbf{u}(0-, t)), \mathbf{u}(0-, t)=\mathbf{u}(\mathbf{w}(0-, t)) .
\end{array}\right.
$$

Then, one can easily check that the analogue of Lemma 7 holds. Provided that the equation of state is a "standard" one, the coupling conditions are again equivalent to (5.3). 


\section{ANALysis of A FLUid MODEL OF PLASMA PHYSICS}

In plasma physics, a classical two-temperature fluid model for a quasi neutral fully ionized plasma composed of ions and electrons is provided by the system of equations

$$
\left\{\begin{array}{l}
\frac{\partial \rho}{\partial t}+\frac{\partial}{\partial x}(\rho v)=0 \\
\frac{\partial}{\partial t}(\rho v)+\frac{\partial}{\partial x}\left(\rho v^{2}\right)+\frac{\partial}{\partial x}\left(p+p_{e}\right)=0 \\
\frac{\partial W}{\partial t}+\frac{\partial}{\partial x}((W+p) v)+v \frac{\partial p_{e}}{\partial x}=S \\
\frac{\partial W_{e}}{\partial t}+\frac{\partial}{\partial x}\left(\left(W_{e}+p_{e}\right) v_{e}\right)-v_{e} \frac{\partial p_{e}}{\partial x}=S_{e}
\end{array}\right.
$$

In (6.1), $\rho$ denotes the mass density of the ion fluid, $v, p$ and $W$ (resp. $v_{e}, p_{e}$ and $W_{e}$ ) denote the mean velocity, the pressure and the energy per unit volume of the ion fluid (resp. the electron fluid). The source terms $S$ and $S_{e}$ take into account effects of collision: Joule effects, relaxation of temperatures. We supplement equations (6.1) with the following thermodynamic closure relations which correspond for the sake of simplicity to a singly ionized monoatomic gas

$$
\left\{\begin{array}{l}
p=\rho R T, W=\rho\left(\frac{3}{2} R T+\frac{1}{2} v^{2}\right), \\
p_{e}=\rho R T_{e}, W_{e}=\rho \frac{3}{2} R T_{e},
\end{array}\right.
$$

where $T$ (resp. $T_{e}$ ) is the ion (electron) temperature and $R$ is the specific gas constant.

In fact, we need an additional closure relation for obtaining a closed system of equations. We then observe that $\rho\left(v-v_{e}\right)$ is proportional to the electric current density. If we specify this current density, this amounts to set

$$
v_{e}=v-\frac{\beta}{\rho}
$$

for some given function $\beta=\beta(x, t)$. One often assumes that this current density is negligible so that $\beta \equiv 0$ and one obtains the usual two-temperature model. In that case, the total energy $W+W_{e}$ satisfies the conservation equation

while $W_{e}$ is solution of

$$
\frac{\partial}{\partial t}\left(W+W_{e}\right)+\frac{\partial}{\partial x}\left(\left(W+W_{e}+\left(p+p_{e}\right) v\right)=S+S_{e}\right.
$$

$$
\frac{\partial W_{e}}{\partial t}+\frac{\partial}{\partial x}\left(\left(W_{e}+p_{e}\right) v\right)-v \frac{\partial p_{e}}{\partial x}=S_{e}
$$

From physical arguments (cf. [34]) or mathematical ones (cf. [7] and the references therein), one knows that the electron pressure $p_{e}$ cannot present any discontinuity so that the electron energy equation makes sense.

The situation is more complex when the current density cannot be neglected. For the sake of simplicity, we will restrict to consider in all the sequel smooth solutions (6.1)-(6.3). More precisely, we will assume that these solutions do not present any shock discontinuity. Then we can put system (6.1)-(6.3) in conservation form (see [30] or [26] for details). Again for simplicity, we will suppose on the one hand that the source terms $S=S_{e}=0$ and on the other hand that $\beta$ is a constant. Introducing "entropies"

$$
s=\frac{p^{\frac{1}{\gamma}}}{\rho}, \quad s_{e}=\frac{p_{e}^{\frac{1}{\gamma}}}{\rho}
$$

with $\gamma=\frac{5}{3}$ for a monoatomic gas, one can easily check that the system (6.1)-(6.3) may be equivalently written in the form

with

$$
\frac{\partial \mathbf{u}}{\partial t}+\frac{\partial}{\partial x} \mathbf{f}(\mathbf{u})=\mathbf{0},
$$

$$
\left\{\begin{array}{l}
\mathbf{u}=\left(\rho, \rho v, \rho s, \rho s_{e}\right)^{T}, \\
\mathbf{f}(\mathbf{u})=\left(\rho v, \rho v^{2}+p+p_{e}, \rho s v, \rho s_{e} v_{e}\right)^{T},
\end{array}\right.
$$




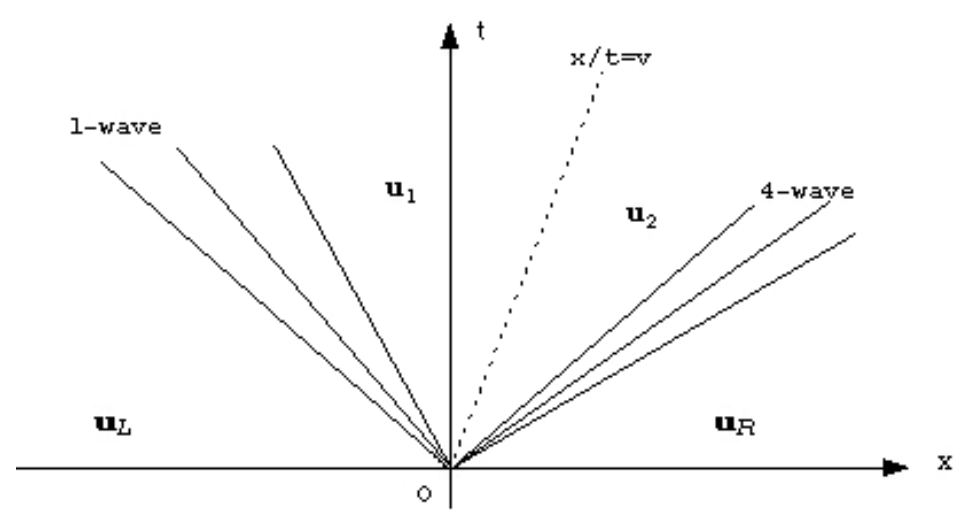

Figure 6.1. Case $\beta=0$, solution of the Riemann problem.

and the closure relation (6.3).

We shall study in Section 7 the coupling of two such systems corresponding to a same $\gamma$-law $(\gamma>1)$ but to different constant current densities, i.e., to different $\beta^{\prime}$ s. As a preliminary step, we solve the Riemann problem for (6.5), (6.6) where $v_{e}$ is defined by (6.3) for some constant $\beta \geq 0$. Let us first discuss the hyperbolicity properties of system (6.5), (6.6). Choosing $\left(\rho, v, p, p_{e}\right)$ as independent variables, this system takes the following nonconservative form:

$$
\left\{\begin{array}{l}
\frac{\partial \rho}{\partial t}+v \frac{\partial \rho}{\partial x}+\rho \frac{\partial v}{\partial x}=0 \\
\frac{\partial v}{\partial t}+v \frac{\partial v}{\partial x}+\frac{1}{\rho} \frac{\partial}{\partial x}\left(p+p_{e}\right)=0 \\
\frac{\partial p}{\partial t}+v \frac{\partial p}{\partial x}+\gamma p \frac{\partial v}{\partial x}=0 \\
\frac{\partial p_{e}}{\partial t}+v_{e} \frac{\partial p_{e}}{\partial x}+\frac{\gamma \beta p_{e}}{\rho^{2}} \frac{\partial \rho}{\partial x}+\gamma p_{e} \frac{\partial v}{\partial x}=0
\end{array}\right.
$$

The eigenvalues $\lambda$ of the associated Jacobian matrix are easily seen to be equal to

$$
\lambda=v, v_{e}, v \pm c, \quad c=\sqrt{\frac{\gamma\left(p+p_{e}\right)}{\rho}} .
$$

Hence the system (6.7) (or (6.5), (6.6)) is strictly hyperbolic if $v_{e} \neq v, v \pm c$, i.e., if $\beta \neq 0, \rho c$. Let us check that it is indeed hyperbolic except for $\beta=\rho c$. The right eigenvectors $\mathbf{r}$ corresponding to the eigenvalues $\lambda$ may be chosen as

$$
\left\{\begin{array}{l}
\lambda=v, \mathbf{r}=\left(\rho, 0,-\gamma p_{e}, \gamma p_{e}\right)^{T} \\
\lambda=v_{e}, \mathbf{r}=\left(\rho,-\frac{\beta}{\rho}, \gamma p, \frac{\beta^{2}}{\rho}-\gamma p\right)^{T} \\
\lambda=v \pm c, \mathbf{r}=\left(\rho, \pm c, \gamma p, \gamma p_{e}\right)^{T}
\end{array}\right.
$$

Thus the characteristic fields associated with $\lambda=v$ and $\lambda=v_{e}$ are linearly degenerate, while those associated with $\lambda=v \pm c$ are genuinely nonlinear except for $v_{e}=v-c$ (or $\beta=\rho c$ ). In this latter case, we obtain a resonant state corresponding to an eigenvalue of multiplicity 2 and an eigenspace of dimension 1 . Observe on the other hand that, for $\beta=0$, the system (6.7) is always hyperbolic.

In fact, for $\beta=0$, the solution of the Riemann problem for (6.5), (6.6) is classical and consists of four constant states separated by a 1 -wave, a double 2,3 -contact discontinuity and a 4 -wave (see Fig. 6.1 ). 
Let us then discuss the case $\beta \neq 0$. We begin by characterizing the discontinuity waves. For a discontinuity wave of velocity $\sigma$ separating two constant states labeled 1 and 2, the Rankine-Hugoniot jump conditions read

$$
\left\{\begin{array}{l}
\sigma[\rho]=[\rho v], \\
\sigma[\rho v]=\left[\rho v^{2}+P\right], \\
\sigma[\rho s]=[\rho s v], \\
\sigma\left[\rho s_{e}\right]=\left[\rho s_{e} v_{e}\right],
\end{array}\right.
$$

where, as usual, for a quantity $\phi,[\phi]$ denotes the jump $\phi_{2}-\phi_{1}$, and we have set

$$
P=p+p_{e}
$$

Since by the first jump relation

$$
M=\rho_{1}\left(v_{1}-\sigma\right)=\rho_{2}\left(v_{2}-\sigma\right)
$$

is constant across the discontinuity, the other jump relations can be equivalently written

$$
\left\{\begin{array}{l}
M=-\frac{[P]}{v v]} \\
M[s]=0, \\
(M-\beta)\left[s_{e}\right]=0 .
\end{array}\right.
$$

We distinguish the three following situations.

(i) $M=0$. This is the case of a material contact discontinuity for which $\sigma=v_{1}=v_{2}$. Then we have

$$
\left\{\begin{array}{l}
{[v]=0} \\
{[P]=0} \\
{\left[s_{e}\right]=0}
\end{array}\right.
$$

(ii) $M=\beta$. This corresponds to the case of the other contact discontinuity for which $\sigma=v_{e, 1}=v_{e, 2}$. We obtain

$$
\left\{\begin{array}{l}
{\left[v_{e}\right]=0,} \\
\beta[v]+[P]=0, \\
{[s]=0 .}
\end{array}\right.
$$

Setting $\tau=\frac{1}{\rho}$, the first two jump relations (6.11) can be also written

$$
\left\{\begin{array}{l}
{[v]=\beta[\tau]} \\
\beta^{2}[\tau]+[P]=0 .
\end{array}\right.
$$

(iii) $M \neq 0, \beta$. This is the case of an "isentropic shock wave". We find

$$
\left\{\begin{array}{l}
\beta M=\frac{[v]}{[\tau]}, \\
M^{2}=\frac{[P]}{[\tau]}, \\
{[s]=\left[s_{e}\right]=0 .}
\end{array}\right.
$$

We next consider rarefaction waves separating two constant states. It is an easy matter to check that the Riemann invariants associated with the characteristic fields

$$
\lambda=v \pm c, \mathbf{r}=\left(\rho, \pm c, \gamma p, \gamma p_{e}\right)^{T}
$$

may be chosen as

$$
s, s_{e}, v \pm \frac{2 c}{\gamma-1}
$$


Then, given a state $\mathbf{u}_{0}$, we look for the states $\mathbf{u}$ that can be connected to $\mathbf{u}_{0}$ by a rarefaction wave associated with $\lambda=v \pm c$. As the corresponding Riemann invariants are constant through the rarefaction wave, we obtain

$$
s=s_{0}, s_{e}=s_{e, 0}, v=v_{0} \mp \frac{2}{\gamma-1}\left(c-c_{0}\right) .
$$

Since

$$
P=\rho^{\gamma}\left(s^{\gamma}+s_{e}^{\gamma}\right)=K_{0} \rho^{\gamma}, \quad K_{0}=s_{0}^{\gamma}+s_{e, 0}^{\gamma},
$$

we have

and therefore

$$
c^{2}=\frac{\gamma P}{\rho}=\gamma K_{0}^{\frac{1}{\gamma}} P^{\frac{\gamma-1}{\gamma}}
$$

$$
v=v_{0} \mp \frac{2 \sqrt{\gamma}}{\gamma-1} K_{0}^{\frac{1}{2 \gamma}}\left(P^{\frac{\gamma-1}{2 \gamma}}-P_{0}^{\frac{\gamma-1}{2 \gamma}}\right) .
$$

Finally, we look for the states $\mathbf{u}$ that can be connected to $\mathbf{u}_{0}$ by an "isentropic shock wave". Using (6.13), we find

with

$$
s=s_{0}, s_{e}=s_{e, 0}, v=v_{0}+M\left(\tau-\tau_{0}\right)
$$

$$
M^{2}=-\frac{P-P_{0}}{\tau-\tau_{0}}
$$

Again, we have (6.14) and therefore

$$
\left[v=v_{0} \pm K_{0}^{\frac{1}{2 \gamma}} \sqrt{\left(P-P_{0}\right)\left(P_{0}^{-\frac{1}{\gamma}}-P^{-\frac{1}{\gamma}}\right)}\right.
$$

Thus setting

$$
\Phi_{0}(P)=\left\{\begin{array}{l}
\frac{2 \sqrt{\gamma}}{\gamma-1} K_{0}^{\frac{1}{2 \gamma}}\left(P^{\frac{\gamma-1}{2 \gamma}}-P_{0}^{\frac{\gamma-1}{2 \gamma}}\right), P \leq P_{0} \\
K_{0}^{\frac{1}{2 \gamma}} \sqrt{\left(P-P_{0}\right)\left(P^{-\frac{1}{\gamma}}-P_{0}^{-\frac{1}{\gamma}}\right)}, P \geq P_{0}
\end{array}, K_{0}=s_{0}^{\gamma}+s_{e, 0}^{\gamma},\right.
$$

and using classical arguments in gas dynamics, we obtain that the states $\mathbf{u}$ that can be connected to $\mathbf{u}_{0}$ on the left by a wave associated with $\lambda=v+c$ (i.e., by a 4 -wave) are given by

$$
\left\{\begin{array}{l}
s=s_{0}, s_{e}=s_{e, 0}, \\
v=v_{0}+\Phi_{0}(P),
\end{array}\right.
$$

while the states $\mathbf{u}$ that can be connected to $\mathbf{u}_{0}$ on the right by a wave associated with $\lambda=v-c$ are given by

$$
\left\{\begin{array}{l}
s=s_{0}, s_{e}=s_{e, 0}, \\
v=v_{0}-\Phi_{0}(P) .
\end{array}\right.
$$

We can now construct the solution of the Riemann problem for the system (6.5), (6.6) corresponding to the initial data

$$
\mathbf{u}_{0}=\left\{\begin{array}{l}
\mathbf{u}_{L}, x<0 \\
\mathbf{u}_{R}, x>0
\end{array}\right.
$$

The construction is different depending on whether $v_{e, L}<v_{L}-c_{L}$ or $v_{e, L}>v_{L}-c_{L}$. For the sake of clarity, we will restrict ourselves to the case

$$
v_{e, L}<v_{L}-c_{L}
$$

which is indeed the situation that we will encounter in Section 7. The case $v_{e, L}>v_{L}-c_{L}$ will be briefly discussed in Appendix B. When (6.19) holds, the solution of the Riemann problem is necessarily of the form below (Fig. 6.2). 


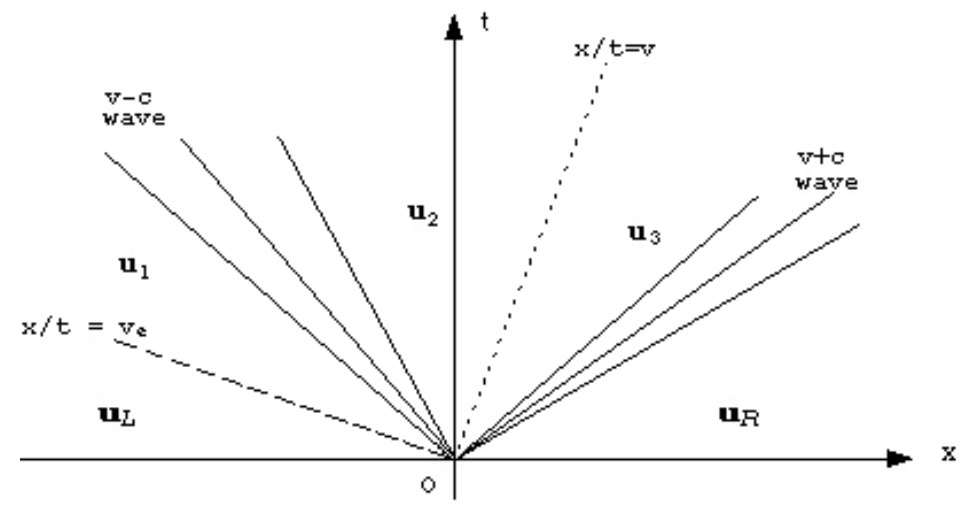

Figure 6.2. Case $\beta \neq 0, v_{e, L}<v_{L}-c_{L}$, solution of the Riemann problem.

Setting

$$
K_{L}=s_{L}^{\gamma}+s_{e, L}^{\gamma}, \quad K_{1}=s_{L}^{\gamma}+s_{e, R}^{\gamma}
$$

we can state

Theorem 4. Assume (6.19). Then, under the condition

$$
P_{L}+\beta^{2}\left(\frac{K_{L}}{P_{L}}\right)^{\frac{1}{\gamma}} \geq(\gamma+1)\left(\beta^{2} \frac{K_{1}^{\frac{1}{\gamma}}}{\gamma}\right)^{\frac{\gamma}{\gamma+1}} \quad \text { if } s_{e, L}<s_{e, R}
$$

(no condition if $s_{e, L} \geq s_{e, R}$ ), the Riemann problem for (6.5), (6.6) has a unique "admissible" solution of the above form.

By admissible solution, we mean a solution which depends continuously on the initial data. Moreover, as in classical gas dynamics, we allow vacuum to appear in order to guarantee the existence of a solution.

Proof. The Rankine-Hugoniot jump conditions (6.10), (6.11) and the relations (6.16), (6.17) yield in that case (using obvious notations)

$$
\begin{gathered}
\left\{\begin{array}{l}
v_{e, 1}=v_{e, L}, \\
\beta\left(v_{1}-v_{L}\right)+P_{1}-P_{L}=0, \\
s_{1}=s_{L}
\end{array}\right. \\
\left\{\begin{array}{l}
v_{2}=v_{1}+\Phi_{1}\left(P_{2}\right) \\
s_{2}=s_{1} \\
s_{e, 2}=s_{e, 1}
\end{array}\right. \\
\left\{\begin{array}{l}
v_{3}=v_{2} \\
P_{3}=P_{2} \\
s_{e, 3}=s_{e, 2}
\end{array}\right.
\end{gathered}
$$

and

We observe that

$$
\left\{\begin{array}{l}
v_{3}=v_{R}+\Phi_{R}\left(P_{3}\right) \\
s_{3}=s_{R} \\
s_{e, 3}=s_{e, R}
\end{array}\right.
$$

$$
\left\{\begin{array}{l}
s_{1}=s_{2}=s_{L}, s_{3}=s_{R} \\
s_{e, 1}=s_{e, 2}=s_{e, 3}=s_{e, R}
\end{array}\right.
$$


As a first consequence, we have indeed

$$
K_{1}=s_{1}^{\gamma}+s_{e, 1}^{\gamma} \text {. }
$$

Let us now check that the conditions (6.22) together with the relation $s_{e, 1}=s_{e, R}$ enable us to determine the state $\mathbf{u}_{1}$ under the condition (6.21). In fact, we need only to find $v_{1}$ and $P_{1}$. Since

$$
\rho_{1}=\left(\frac{P_{1}}{K_{1}}\right)^{\frac{1}{\gamma}}, \rho_{L}=\left(\frac{P_{L}}{K_{L}}\right)^{\frac{1}{\gamma}},
$$

the first two conditions (6.22) give respectively

$$
\left\{\begin{array}{l}
v_{1}=v_{L}+\beta\left(\left(\frac{K_{1}}{P_{1}}\right)^{\frac{1}{\gamma}}-\left(\frac{K_{L}}{P_{L}}\right)^{\frac{1}{\gamma}}\right), \\
v_{1}=v_{L}+\frac{1}{\beta}\left(P_{L}-P_{1}\right) .
\end{array}\right.
$$

Eliminating $v_{1}$ between the above equations yields

$$
P_{1}+\beta^{2}\left(\frac{K_{1}}{P_{1}}\right)^{\frac{1}{\gamma}}=P_{L}+\beta^{2}\left(\frac{K_{L}}{P_{L}}\right)^{\frac{1}{\gamma}} .
$$

Then we use the following result whose proof is given in Appendix A.

Lemma 8. Given $a>0$, we set

$$
f(\xi ; a)=\xi+\frac{a}{\xi^{\frac{1}{\gamma}}}, \xi>0,
$$

and we consider the equation

$$
f(\eta(\xi) ; b)=f(\xi ; a)
$$

where $b>0$ is a parameter. Then if $b>a$, equation $(6.25)$ has at least one solution $\eta(\xi)=\eta(\xi ; b)$ if and only if

$$
f(\xi ; a) \geq(\gamma+1)\left(\frac{b}{\gamma}\right)^{\frac{\gamma}{\gamma+1}}
$$

it has exactly one solution if the equality holds in (6.26) and two solutions if the strict inequality holds; if $b \leq a$, equation (6.25) has two solutions.

In both cases, among the possible solutions of (6.25), there exists only one solution which satisfies

$$
\eta(\xi ; b) \rightarrow \xi \text { as } b \rightarrow a
$$

We then apply Lemma 8 with $a=\beta^{2} K_{L}^{\frac{1}{\gamma}}$ and $b=\beta^{2} K_{1}^{\frac{1}{\gamma}}$ for $s_{e, R}>s_{e, L}(i . e ., b>a)$, a necessary and sufficient condition for equation (6.23) to have at least one solution is given by (6.21), while for for $s_{e, R} \leq s_{e, L}$ (i.e., $b \leq a)$, equation (6.23) has always two solutions. In any case, we select the admissible solution as the unique one which satisfies

$$
P_{1} \rightarrow P_{2} \text { as } s_{e, R} \rightarrow s_{e, L}
$$

This determines the state $\mathbf{u}_{1}$ under the condition (6.21).

Next setting

$$
v_{*}=v_{2}=v_{3}, \quad P_{*}=P_{2}=P_{3},
$$

it remains only to find the pair $\left(v_{*}, P_{*}\right)$ solution of the system

$$
\left\{\begin{array}{l}
v_{*}=v_{1}-\Phi_{1}\left(P_{*}\right) \\
v_{*}=v_{R}+\Phi_{R}\left(P_{*}\right),
\end{array}\right.
$$


or equivalently to find the intersection in the $(v, P)$-plane of the 2 and 4 -wave curves. These curves indeed intersect at a unique point if and only if

$$
v_{1}-\Phi_{1}(0) \geq v_{R}+\Phi_{R}(0)
$$

or if and only if

$$
v_{R}-v_{1} \leq \frac{2 \sqrt{\gamma}}{\gamma-1}\left(K_{R}^{\frac{1}{2 \gamma}} P_{R}^{\frac{\gamma-1}{2 \gamma}}-K_{1}^{\frac{1}{2 \gamma}} P_{1}^{\frac{\gamma-1}{2 \gamma}}\right)
$$

When this latter condition does not hold, vacuum appears exactly as in the solution of the Riemann problem for the classical gas dynamics equations.

For $s_{e, L} \geq s_{e, R}$, Theorem 4 provides a global existence and uniqueness result for the solution of the Riemann problem. For $s_{e, L}<s_{e, R}$, it remains to check the condition (6.21) where resonance does not occur. Let us notice that this condition trivially holds for $\beta$ small enough or $\beta$ large enough (due to the fact that $1<2 \gamma /(\gamma+1)<2$ ), i.e., for an electric current density small enough or large enough. Indeed, in [30], we have not met physical situations where resonance occurs. Therefore we have not tried to deal with resonance problems which are beyond the scope of this paper and we refer to $[21,24]$ for the study of resonance. See however Remark 9.

\section{The COUPLING OF TWO PLASMA FLUID MODELS}

We want to couple two plasma models of the form (6.5), (6.6) corresponding to different $\beta$ 's, namely $\beta_{L}=\beta, \beta_{R}=0$ (cf. [30] for a physical derivation of such a coupled problem). Remark that only the 4th equation of conservation of the electron entropy changes at the interface $x=0$. This equation reads

$$
\frac{\partial}{\partial t}\left(\rho s_{e}\right)+\frac{\partial}{\partial x}\left(\rho s_{e} v_{e}\right)=0
$$

with

$$
v_{e}= \begin{cases}v-\frac{\beta}{\rho}, & x<0 \\ v, & x>0\end{cases}
$$

Thus we have here

$$
\begin{aligned}
& \mathbf{u}=\left(\rho, \rho v, \rho s, \rho s_{e}\right)^{T}, \\
& \mathbf{f}_{L}(\mathbf{u})=\left(\rho v, \rho v^{2}+P, \rho s v, \rho s_{e} v-\beta s_{e}\right)^{T}, \\
& \mathbf{f}_{R}(\mathbf{u})=\left(\rho v, \rho v^{2}+P, \rho s v, \rho s_{e} v\right)^{T},
\end{aligned}
$$

where $P=p+p_{e}=(\rho s)^{\gamma}+\left(\rho s_{e}\right)^{\gamma}(\gamma=5 / 3$ for our physical application). The first step in this coupling problem consists in expressing the conditions (2.6). It is out of scope to treat all the possible coupling situations according to the sign of the eigenvalues. In fact, we are interested in the situation where $v_{e}<v-c<0<v<v+c$. In the linear case with constant coefficients analyzed in Section 3, this case would correspond to the case $p=4, q_{L}=2, q_{R}=1$. In such a situation, we know from Theorem 3 that the solution of the coupled problem is not unique. We will see that this is also true for the nonlinear coupled problem.

Assume therefore that the states $\mathbf{u}(0-, t)$ and $\mathbf{u}(0+, t)$ satisfy

$$
\left\{\begin{array}{l}
v_{e}(0-, t)=\left(v-\frac{\beta}{\rho}\right)(0-, t)<(v-c)(0-, t)<0<v(0-, t)<(v+c)(0-, t), \\
(v-c)(0+, t)<0<v_{e}(0+, t)=v(0+, t)<(v+c)(0+, t) .
\end{array}\right.
$$

Lemma 9. Under the hypotheses (7.3), the coupling conditions (2.6) are equivalent to

$$
\mathbf{u}(0-, t)=\mathbf{u}(0+, t)
$$


Proof. Let us first discuss the condition

$$
\mathbf{u}(0+) \in \mathcal{O}_{R}(\mathbf{u}(0-))=\left\{\mathbf{W}_{R}\left(0+; \mathbf{u}(0-), \mathbf{u}_{R}\right) ; \mathbf{u}_{R} \in \Omega\right\}
$$

where, for the sake of simplicity, we have dropped the dependence of $\mathbf{u}(0 \pm, t)$ on $t$. Condition (7.5) yields the following situation (Fig. 7.1) where the states $\mathbf{u}(0-)$ and $\mathbf{u}(0+)$ are connected by a $1-R$ wave $(i . e$., a $1-$ wave associated with the flux function $\mathbf{f}_{R}$ ). We have therefore

$$
\left\{\begin{array}{l}
v(0+)=v(0-)-\Phi_{\mathbf{u}_{0}(0-)}(P(0+)) \\
s(0+)=s(0-) \\
s_{e}(0+)=s_{e}(0-)
\end{array}\right.
$$

Let us next consider the condition

$$
\mathbf{u}(0-) \in \mathcal{O}_{L}(\mathbf{u}(0+))=\left\{\mathbf{W}_{L}\left(0-; \mathbf{u}(0+), \mathbf{u}_{L}\right) ; \mathbf{u}_{L} \in \Omega\right\}
$$

Applying the results of Section 6, we obtain here the situation described in Figure 7.2.

The states $\mathbf{u}^{*}$ and $\mathbf{u}(0+)$ are connected by a $4-L$ wave, while the states $\mathbf{u}(0-)$ and $\mathbf{u}^{*}$ are connected by a $3-L$ contact discontinuity. Hence we find

$$
\left\{\begin{array}{l}
v^{*}=v(0+)+\Phi_{\mathbf{u}_{0}(0+)}\left(P^{*}\right) \\
s^{*}=s(0+) \\
s_{e}^{*}=s_{e}(0+)
\end{array}\right.
$$

and

$$
\left\{\begin{array}{l}
v(0-)=v^{*} \\
P(0-)=P^{*} \\
s_{e}(0-)=s_{e}^{*}
\end{array}\right.
$$

so that

$$
\left\{\begin{array}{l}
v(0-)=v(0+)+\Phi_{\mathbf{u}_{0}(0+)}(P(0-)) \\
P(0-)=P^{*} \\
s_{e}(0-)=s_{e}(0+) .
\end{array}\right.
$$

Observe that the function $\Phi_{\mathbf{u}}$ is in fact the same for both systems. We thus have

$$
\left\{\begin{array}{l}
v(0+)=v(0-)-\Phi_{\mathbf{u}_{0}(0-)}(P(0+)), \\
v(0-)=v(0+)+\Phi_{\mathbf{u}_{0}(0+)}(P(0-)) .
\end{array}\right.
$$

Since the corresponding curves in the $(v, P)$-plane can intersect at one point at most, we get

$$
\left\{\begin{array}{l}
v(0-)=v(0+) \\
P(0-)=P(0+)
\end{array}\right.
$$

Together with the last two equations (7.6), this yields the continuity constraint (7.4).

We are now able to solve the Riemann problem for the coupled system: given two states $\mathbf{u}_{L}$ and $\mathbf{u}_{R}$ satisfying $(c f .(7.3))$

$$
\left\{\begin{array}{l}
v_{e, L}<v_{L}-c_{L}<0<v_{L}<v_{L}+c_{L} \\
v_{R}-c_{R}<0<v_{e, R}=v_{R}<v_{R}+c_{R}
\end{array}\right.
$$




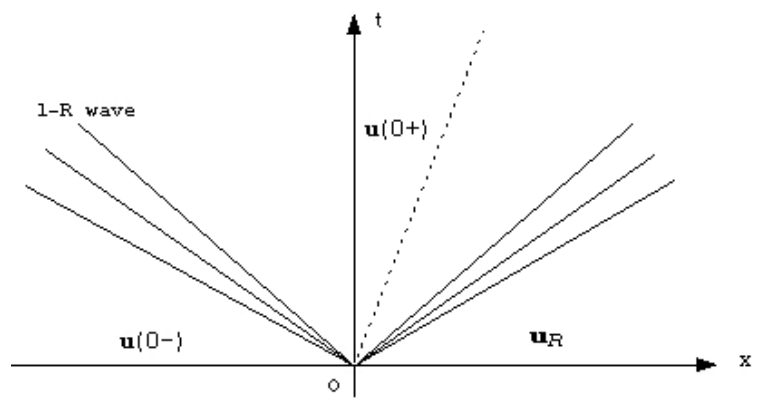

FiguRE 7.1. $\mathbf{u}(0+) \in \mathcal{O}_{R}(\mathbf{u}(0-))$.

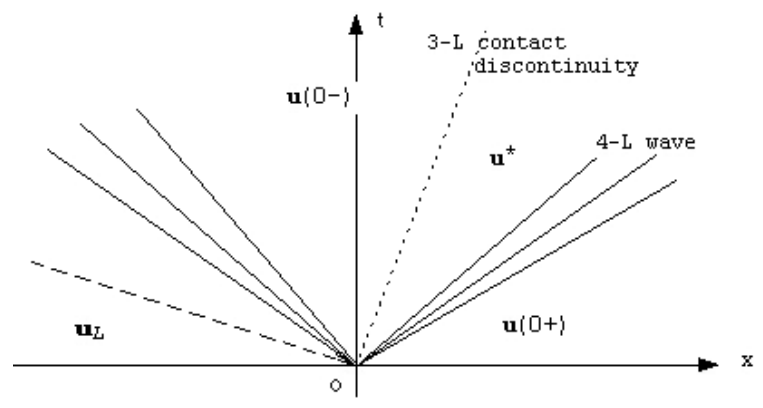

Figure 7.2. $\mathbf{u}(0-) \in \mathcal{O}_{L}(\mathbf{u}(0+))$.

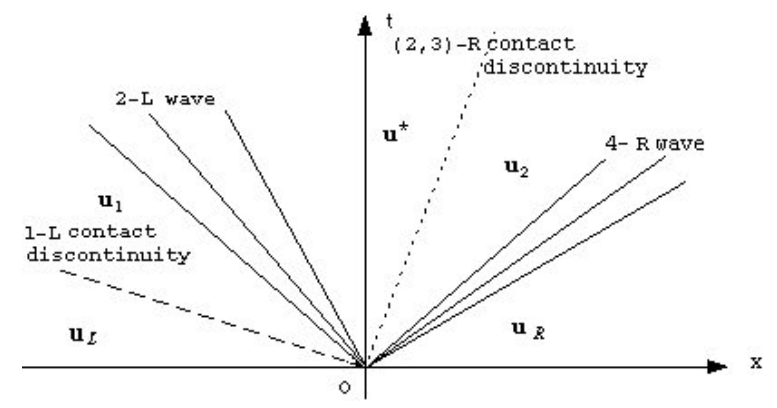

FigURE 7.3. Self-similar solution of the coupled system (7.10).

we consider the coupled problem

$$
\left\{\begin{array}{l}
\frac{\partial \mathbf{u}}{\partial t}+\frac{\partial}{\partial x} \mathbf{f}_{L}(\mathbf{u})=\mathbf{0}, \quad x<0, t>0 \\
\frac{\partial \mathbf{u}}{\partial t}+\frac{\partial}{\partial x} \mathbf{f}_{R}(\mathbf{u})=\mathbf{0}, \quad x>0, t>0 \\
\mathbf{u}(x, 0)= \begin{cases}\mathbf{u}_{L}, & x<0 \\
\mathbf{u}_{R}, & x>0\end{cases} \\
\mathbf{u}(0-, t)=\mathbf{u}(0+, t), \quad t>0
\end{array}\right.
$$

Let us show that, under the conditions (7.9), problem (7.10) possesses (at least) a one-parameter family of self-similar solutions. We first observe that, in the coupling of two linear hyperbolic systems with constant coefficients considered in Section 3 and for $q_{L}>q_{R}$, the coupled Riemann problem possesses an affine variety of dimension $q_{L}-q_{R}$ of solutions consisting of $p+1+q_{L}-q_{R}$ constant states separated by $q_{L} L$-waves with negative speeds and $p-q_{R} R$-waves with positive speeds. Hence, if we use a linearized approach and consider the coupling of the linearized plasma systems at $\mathbf{u}_{L}$ and $\mathbf{u}_{R}$ respectively, we have $p=4, q_{L}=2, q_{R}=1$ and we may expect a one-parameter family of five waves solutions to appear. But since $\lambda_{2}^{R}=\lambda_{3}^{R}$, we have a double wave and the solution of the corresponding coupled Riemann problem consists of five constant states separated by two $L$-waves with negative speeds and two $R$-waves with positive speeds. Now for our coupled Riemann problem (7.10), it seems fairly natural to look for a solution of the form depicted in Figure 7.3.

Thus, we expect the second wave to be a $L$-wave with negative speed (as in Fig. 7.3). However, the problem is nonlinear and there is no evidence that this holds true. In fact, as we shall see it in the proof of Theorem 5 , the second wave is allowed to have positive speeds: this follows from the fact that the $2-L$ wave and the $1-R$ wave are characterized in the same way. 
We can state

Theorem 5. Assume the conditions (7.9). Then the coupled Riemann problem (7.10) admits a one-parameter family of self-similar solutions parameterized by $s_{e}^{*} \in\left[0, s_{e, \max }^{*}\right]$ where

$$
s_{e, \max }^{*}=\left\{\left(\frac{\gamma}{\beta^{2}}\right)^{\gamma}\left(\frac{1}{\gamma+1}\left(P_{L}+\beta^{2}\left(\frac{K_{L}}{P_{L}}\right)^{\frac{1}{\gamma}}\right)\right)^{\gamma+1}-s_{L}^{\gamma}\right\}^{\frac{1}{\gamma}} .
$$

Proof. The proof is fairly similar to that of Theorem 4. We look for a solution consisting of five constant states. First the states $\mathbf{u}_{L}$ and $\mathbf{u}_{1}$ are connected by a $1-L$ wave so that

$$
\left\{\begin{array}{l}
v_{e, 1}=v_{e, L} \\
\beta\left(v_{1}-v_{L}\right)+P_{1}-P_{L}=0 \\
s_{1}=s_{L}
\end{array}\right.
$$

If we assume that the states $\mathbf{u}_{1}$ and $\mathbf{u}^{*}$ are connected by a $2-L$ wave we have

$$
\left\{\begin{array}{l}
v^{*}=v_{1}-\Phi_{1}\left(P^{*}\right) \\
s^{*}=s_{1} \\
s_{e}^{*}=s_{e, 1}
\end{array}\right.
$$

In fact, since the function $\Phi_{0}$ defined by (6.15) does not depend on $\beta$, these relations only mean that the states $\mathbf{u}_{1}$ and $\mathbf{u}^{*}$ are connected by a wave associated with the eigenvalue $v-c$. Next, assuming that the states $\mathbf{u}^{*}$ and $\mathbf{u}_{2}$ are connected by a double $(2,3)-R$ contact discontinuity while the states $\mathbf{u}_{2}$ and $\mathbf{u}_{R}$ are connected by a $4-R$ wave, we obtain

and

$$
\left\{\begin{array}{l}
v_{2}=v^{*} \\
P_{2}=P^{*}
\end{array}\right.
$$

$$
\left\{\begin{array}{l}
v_{2}=v_{R}+\Phi_{R}\left(P_{2}\right) \\
s_{2}=s_{R} \\
s_{e, 2}=s_{e, R}
\end{array}\right.
$$

We thus obtain 11 scalar equations in 12 unknowns corresponding to the states $\mathbf{u}_{1}, \mathbf{u}^{*}$ and $\mathbf{u}_{2}$. Then, for any choice of $s_{e}^{*}$ in the interval $\left[0, s_{e, \text { max }}^{*}\right.$, we next check that the above equations define a self-similar solution of the Riemann problem in a unique way. As in the proof of Theorem 4, the relations (7.12) together with the constraint $s_{e}^{*}=s_{e, 1}$ determine the state $\mathbf{u}_{1}$. Indeed, on the one-hand, $P_{1}$ satisfies equation (6.23) with

$$
K_{1}=s_{L}^{\gamma}+\left(s_{e}^{*}\right)^{\gamma}
$$

By Lemma 8, this equation has a unique admissible solution provided that either

$$
s_{e}^{*} \leq s_{e, L},
$$

or, in the case $s_{e}^{*}>s_{e, L}$, if

$$
P_{L}+\beta^{2}\left(\frac{K_{L}}{P_{L}}\right)^{\frac{1}{\gamma}} \geq(\gamma+1)\left(\beta^{2} \frac{K_{1}^{\frac{1}{\gamma}}}{\gamma}\right)^{\frac{\gamma}{\gamma+1}}
$$

Since by Hölder's inequality

$$
P_{L}+\beta^{2}\left(\frac{K_{L}}{P_{L}}\right)^{\frac{1}{\gamma}} \geq(\gamma+1)\left(\beta^{2} \frac{K_{L}^{\frac{1}{\gamma}}}{\gamma}\right)^{\frac{\gamma}{\gamma+1}}
$$


the above solvability conditions reduce in fact to (7.13) which reads equivalently

$$
s_{e}^{*} \leq s_{e, \max }^{*}
$$

where $s_{e, \max }^{*}$ is defined by (7.11). On the other hand, we have

$$
\left\{\begin{array}{l}
v_{1}=v_{L}+\frac{1}{\beta}\left(P_{1}-P_{L}\right) \\
s_{1}=s_{L} \\
s_{e, 1}=s_{e}^{*}
\end{array}\right.
$$

The state $\mathbf{u}_{1}$ being obtained, it remains only to determine the pair $\left(v^{*}, P^{*}\right)$ solution of the system

$$
\left\{\begin{array}{l}
v^{*}=v_{1}-\Phi_{1}\left(P^{*}\right) \\
v^{*}=v_{R}+\Phi_{R}\left(P^{*}\right)
\end{array}\right.
$$

or equivalently to solve a classical Riemann problem for the gas dynamics equations which has a unique solution (provided that we allow vacuum to appear).

Remark 7. The fact that the second wave may have a positive or a vanishing speed, also follows from a (heuristic) linearized approach. On the one hand, if we have $(v-c)(0-)>0,(7.3)$ has to be replaced by

$$
\left\{\begin{array}{l}
v_{e}(0-, t)<0<(v-c)(0-, t)<v(0-, t)<(v+c)(0-, t) \\
0<(v-c)(0+, t)<v_{e}(0+, t)=v(0+, t)<(v+c)(0+, t)
\end{array}\right.
$$

and it is straightforward to check that the coupling conditions $(2.6)$ are again equivalent to $\mathbf{u}(0-, t)=\mathbf{u}(0+, t)$. Thus, linearizing the two systems at the same state $\mathbf{u}(0, t)$, we obtain $q_{L}=1>q_{R}=0$, and therefore a one-parameter family of solutions of the Riemann problem whose second wave has positive velocities. If, on the other hand, we have $(v-c)(0, t)=0$, then linearizing the two systems at $\mathbf{u}(0, t)$ gives now $q_{L}=1=q_{R}$. However, since $\ell_{R, 1}$ and $\ell_{L, 2}$ coincide (they correspond to the same eigenvalue $v-c$ ), the uniqueness condition of Theorem $1\left(\left\{\ell_{L, 1}, \ell_{R, 2}, \ell_{R, 3}, \ell_{R, 4}\right\}\right.$ and $\left\{\ell_{R, 1}, \ell_{L, 2}, \ell_{L, 3}, \ldots \ell_{L, 4}\right\}$ are two bases of $\left.\mathbb{R}^{4}\right)$ is not met and again the solutions of the Riemann problem form a one-parameter family.

It remains to know the parameter $s_{e}^{*}$ in order to specify the solution of the Riemann problem (7.10). We first observe that choosing $s_{e}^{*}=s_{e, L}$ amounts to solve an uncoupled Riemann problem, namely the Riemann problem for the usual gas dynamics equations. Indeed, for $s_{e}^{*}=s_{e, L}, P_{1}$ satisfies equation (6.23) with $K_{1}=s_{L}^{\gamma}+s_{e, L}^{\gamma}=K_{L}$ so that $P_{1}=P_{L}$ and therefore $v_{1}=v_{L}$. Hence, we obtain $\mathbf{u}_{1}=\mathbf{u}_{L}$ and our assertion follows.

We will see in the next section that various numerical methods of upwind type approximate solutions of (7.10) given by Theorem 5 and corresponding to a value of the parameter $s_{e}^{*}$ depending on the equation of state, i.e., on the adiabatic exponent $\gamma$, on the initial conditions and also "slightly" on the numerical scheme and the CFL.

Remark 8. It is an open question to know whether all the solutions of problem (7.10) are given by Theorem 5 or if there exist other solutions which exhibit a fewer number of waves. However, we have not observed such solutions numerically.

Remark 9. Since by (7.9), $v_{e}$ jumps over $v-c$ when passing from the left state $\mathbf{u}_{L}$ to the right state $\mathbf{u}_{R}$, the self-similar solutions given by Theorem 5 could eventually exhibit a resonant state. This is not the case, at least in numerical computations: the first eigenvalue of the Jacobian matrix jumps over the second one at the interface so that the resonant state is not observed. On the other hand, when the coupling is ensured through a regularization of a flux function, the resonant state is indeed attained and the numerical solution presents a non physical peak close to the interface. In fact, our coupling method seems to avoid resonance problems at the interface. This point deserves more investigation in order to see if this property could be general. 


\section{The Numerical COUPLing of THE TWO Plasma Fluid MODELS}

We have already proved in the scalar case (see [17]) that we need to use an upwind scheme, whose prototype is the Godunov method, in order to ensure adequate numerical coupling of the two models. On the other hand, since in the situation (7.3) we have shown in the previous section that the coupled Cauchy problem possesses in general a continuum of solutions, a natural question arises: does the "converged" numerical solution depend on the chosen upwind scheme? We thus introduce several upwind schemes for solving the coupled problem.

We first consider the simplest Godunov-type method, namely the HLLE method [20] which corresponds to the numerical fluxes

$$
\left\{\begin{aligned}
\mathbf{g}_{\alpha, j}= & \mathbf{g}_{\alpha}\left(\mathbf{u}_{j-1 / 2}, \mathbf{u}_{j+1 / 2}\right), \quad \alpha=L, R, \\
= & \frac{1}{b_{\alpha, j}^{+}-b_{\alpha, j}^{-}}\left(b_{\alpha, j}^{+} \mathbf{f}_{\alpha}\left(\mathbf{u}_{j-1 / 2}\right)-b_{\alpha, j}^{-} \mathbf{f}_{\alpha}\left(\mathbf{u}_{j+1 / 2}\right)\right) \\
& +\frac{1}{b_{\alpha, j}^{+}-b_{\alpha, j}^{-}} b_{\alpha, j}^{-} b_{\alpha, j}^{+}\left(\mathbf{u}_{j+1 / 2}-\mathbf{u}_{j-1 / 2}\right),
\end{aligned}\right.
$$

where the $b_{\alpha, j}^{ \pm}$are chosen in such a way that the HLLE scheme is entropy preserving, namely

$$
\left\{\begin{array}{l}
b_{L, j}^{-}=\min \left(v_{j-1 / 2}-c_{j-1 / 2}, v_{e, j-1 / 2}\right), b_{L, j}^{+}=v_{j+1 / 2}+c_{j+1 / 2} \\
b_{R, j}^{-}=v_{j-1 / 2}-c_{j-\frac{1}{2}}, b_{R, j}^{+}=v_{j+1 / 2}+c_{j+1 / 2}
\end{array}\right.
$$

and we use the associated coupled scheme (2.8). Next we use the second order MUSCL method constructed from the HLLE method.

\section{Defining}

$$
\left\{\begin{array}{l}
\mathbf{u}_{j}^{+}=\mathbf{u}_{j+1 / 2}-\frac{\Delta x}{2} \mathbf{s}_{j+1 / 2}, \\
\mathbf{u}_{j}^{-}=\mathbf{u}_{j-1 / 2}+\frac{\Delta x}{2} \mathbf{s}_{j-1 / 2},
\end{array}\right.
$$

where $\mathbf{s}_{j-1 / 2}$ is the slope in the cell $j+1 / 2$ obtained by the min-mod procedure (for instance), we set

$$
\left\{\begin{array}{l}
\overline{\mathbf{u}}_{j}^{+}=\mathbf{u}_{j}^{+}-\frac{\Delta t}{2 \Delta x}\left(\mathbf{f}\left(\mathbf{u}_{j+1}^{-}\right)-\mathbf{f}\left(\mathbf{u}_{j}^{+}\right)\right) \\
\overline{\mathbf{u}}_{j}^{-}=\mathbf{u}_{j}^{-}-\frac{\Delta t}{2 \Delta x}\left(\mathbf{f}\left(\mathbf{u}_{j}^{-}\right)-\mathbf{f}\left(\mathbf{u}_{j-1}^{+}\right)\right.
\end{array}\right.
$$

and we take as numerical fluxes

$$
\left\{\begin{aligned}
\overline{\mathbf{g}}_{\alpha, j}= & \overline{\mathbf{g}}_{\alpha}\left(\overline{\mathbf{u}}_{j}^{-}, \overline{\mathbf{u}}_{j}^{+}\right), \quad \alpha=L, R \\
= & \frac{1}{\bar{b}_{\alpha, j}^{+}-\bar{b}_{\alpha, j}^{-}}\left(\bar{b}_{\alpha, j}^{+} \mathbf{f}_{\alpha}\left(\overline{\mathbf{u}}_{j}^{-}\right)-\bar{b}_{\alpha, j}^{-} \mathbf{f}_{\alpha}\left(\overline{\mathbf{u}}_{j}^{+}\right)\right) \\
& +\frac{1}{b_{\alpha, j}^{+}-\bar{b}_{\alpha, j}^{-}} \bar{b}_{\alpha, j}^{-} \bar{b}_{\alpha, j}^{+}\left(\overline{\mathbf{u}}_{j}^{+}-\overline{\mathbf{u}}_{j}^{-}\right)
\end{aligned}\right.
$$

with

$$
\left\{\begin{array}{l}
\bar{b}_{L, j}^{-}=\min \left(\bar{v}_{j}^{-}-\bar{c}_{j}^{-}, \bar{v}_{e, j}^{-}\right), \quad \bar{b}_{L, j}^{+}=\bar{v}_{j}^{+}+\bar{c}_{j}^{+}, \\
\bar{b}_{R, j}^{-}=\bar{v}_{j}^{-}-\bar{c}_{j}^{-}, \quad \bar{b}_{R, j}^{+}=\bar{v}_{j}^{+}+\bar{c}_{j}^{+} .
\end{array}\right.
$$

As it is well known, the HLLE method does not resolve accurately contact discontinuities. This could appear here as a major drawback of the method since the discontinuities of $s_{e}$ indeed arise at contact discontinuities. Hence we have considered a more elaborate scheme based on a Roe-type solver. In fact, it is easy to construct such a Roe solver in the framework of Lagrange-projection methods (see for instance [16]) which are commonly used in the numerical simulation of physically complex problems. In Lagrange coordinates, the plasma fluid model (6.5), (6.6) takes the simple form

with

$$
\frac{\partial \mathbf{w}}{\partial t}+\frac{\partial}{\partial m} \mathbf{h}(\mathbf{w})=\mathbf{0},
$$

$$
\left\{\begin{array}{l}
\mathbf{w}=\left(\tau, v, s_{e}, s\right)^{T} \\
\mathbf{h}(\mathbf{w})=\left(-u, p+p_{e},-\beta s_{e}, 0\right)^{T}
\end{array}\right.
$$


where $\tau=\frac{1}{\rho}$ and $m$ is a mass variable such that $\mathrm{d} m=\rho_{0} \mathrm{~d} x$. Given two states $\mathbf{w}_{1}$ and $\mathbf{w}_{2}$, we set $\Delta \mathbf{w}=\mathbf{w}_{2}-\mathbf{w}_{1}$. In order to obtain a Roe matrix for the system (8.7), we follow the approach of Gallice [12]. It is enough to postulate two relations of the form

$$
\left\{\begin{array}{l}
\Delta p_{e}=\chi_{e} \Delta \tau+\kappa_{e} \Delta s_{e} \\
\Delta p=\chi \Delta \tau+\kappa \Delta s
\end{array}\right.
$$

where $\chi_{e}$ and $\kappa_{e}$ (resp. $\chi$ and $\kappa$ ) are approximations of $\frac{\partial p_{e}}{\partial \tau}=-\frac{\gamma p_{e}}{\tau}$ and $\frac{\partial p_{e}}{\partial s_{e}}=\frac{\gamma p_{e}}{s_{e}}$ (resp. of $\frac{\partial p}{\partial \tau}=-\frac{\gamma p}{\tau}$ and $\frac{\partial p}{\partial s}=\frac{\gamma p}{s}$ ) between the two states $\mathbf{w}_{1}$ and $\mathbf{w}_{2}$. Then the matrix

$$
\mathbf{B}=\mathbf{B}\left(\mathbf{w}_{1}, \mathbf{w}_{2}\right)=\left(\begin{array}{cccc}
0 & -1 & 0 & 0 \\
\chi+\chi_{e} & 0 & \kappa_{e} & \kappa \\
0 & 0 & -\beta & 0 \\
0 & 0 & 0 & 0
\end{array}\right)
$$

satisfies

$$
\Delta \mathbf{h}(\mathbf{w})=\mathbf{B} \cdot \Delta \mathbf{w} .
$$

The numerical flux $\mathbf{k}=\mathbf{k}\left(\mathbf{w}_{1}, \mathbf{w}_{2}\right)$ of the associated Roe method is easily computed

$$
\mathbf{k}=\frac{1}{2}\left(\mathbf{h}\left(\mathbf{w}_{1}\right)+\mathbf{h}\left(\mathbf{w}_{2}\right)-|\mathbf{B}| \Delta \mathbf{w}\right)=\left(\begin{array}{c}
-\bar{v}+\frac{1}{2 C} \Delta\left(p+p_{e}\right)-\frac{\beta \kappa_{e}}{2 C(\beta+C)} \Delta s_{e} \\
\overline{p+p_{e}}-\frac{C}{2} \Delta v+\frac{\beta \kappa_{e}}{2(\beta+C)} \Delta s_{e} \\
-\beta s_{e, 2} \\
0
\end{array}\right)
$$

where $\bar{\phi}=\frac{1}{2}\left(\phi_{1}+\phi_{2}\right)$ and

$$
C=\sqrt{-\left(\chi+\chi_{e}\right)}
$$

is an approximation of the Lagrangian sound speed $C_{L}=\sqrt{-\frac{\partial}{\partial \tau}\left(p+p_{e}\right)}$. Note that we need only to determine $C$ and $\kappa_{e}$ for specifying the numerical scheme. The choices of $C$ and $\kappa_{e}$ are arbitrary to a certain extent at the only restriction that they have to meet accuracy and entropy constraints $(c f .[8,12])$. Here we have chosen (cf. [29])

$$
C=\gamma \frac{\overline{p+p_{e}}}{\tau}, \kappa_{e}=\frac{\gamma \overline{p_{e}}}{\overline{s_{e}}} .
$$

The Lagrange-projection Roe scheme for the coupled plasma problem is defined as follows. Starting from the cell quantities $\rho_{j+1 / 2}^{n}, v_{j+1 / 2}^{n}, s_{e, j+1 / 2}^{n}, s_{j+1 / 2}^{n}, j \in \mathbb{Z}$, at time $t_{n}$, we compute

$$
p_{e, j+1 / 2}^{n}=\left(\rho_{j+1 / 2}^{n} s_{e, j+1 / 2}^{n}\right)^{\gamma}, p_{j+1 / 2}^{n}=\left(\rho_{j+1 / 2}^{n} s_{j+1 / 2}^{n}\right)^{\gamma}, P_{j+1 / 2}^{n}=\left(p+p_{e}\right)_{j+1 / 2}^{n},
$$

and we define quantities at the cell edges $x_{j}=j \Delta x$ : for $j \leq 0(c f$. (8.11))

$$
\left\{\begin{array}{l}
v_{j}^{n}=\frac{1}{2}\left(v_{j-1 / 2}^{n}+v_{j+1 / 2}^{n}\right)-\frac{1}{2 C_{j}^{n}}\left(P_{j+1 / 2}^{n}-P_{j-1 / 2}^{n}-\frac{\beta \kappa_{e, j}^{n}}{\beta+C_{n}^{n}}\left(s_{e, j+1 / 2}^{n}-s_{e, j-1 / 2}^{n}\right)\right) \\
P_{j}^{n}=\frac{1}{2}\left(P_{j-1 / 2}^{n}+P_{j+1 / 2}^{n}\right)-\frac{C_{j}^{n}}{2}\left(v_{j+1 / 2}^{n}-v_{j-1 / 2}^{n}\right)+\frac{\beta \kappa_{e, j}^{n}}{2\left(\beta+C_{j}^{n}\right)}\left(s_{e, j+1 / 2}^{n}-s_{e, j-1 / 2}^{n}\right) \\
s_{e, j}^{n}=s_{e, j+1 / 2}^{n}
\end{array}\right.
$$

and for $j \geq 0$

$$
\left\{\begin{array}{l}
v_{j}^{n}=\frac{1}{2}\left(v_{j-1 / 2}^{n}+v_{j+1 / 2}^{n}\right)-\frac{1}{2 C_{j}^{n}}\left(P_{j+1 / 2}^{n}-P_{j-1 / 2}^{n}\right) \\
P_{j}^{n}=\frac{1}{2}\left(P_{j-1 / 2}^{n}+P_{j+1 / 2}^{n}\right)-\frac{C_{j}^{n}}{2}\left(v_{j+1 / 2}^{n}-v_{j-1 / 2}^{n}\right)
\end{array}\right.
$$



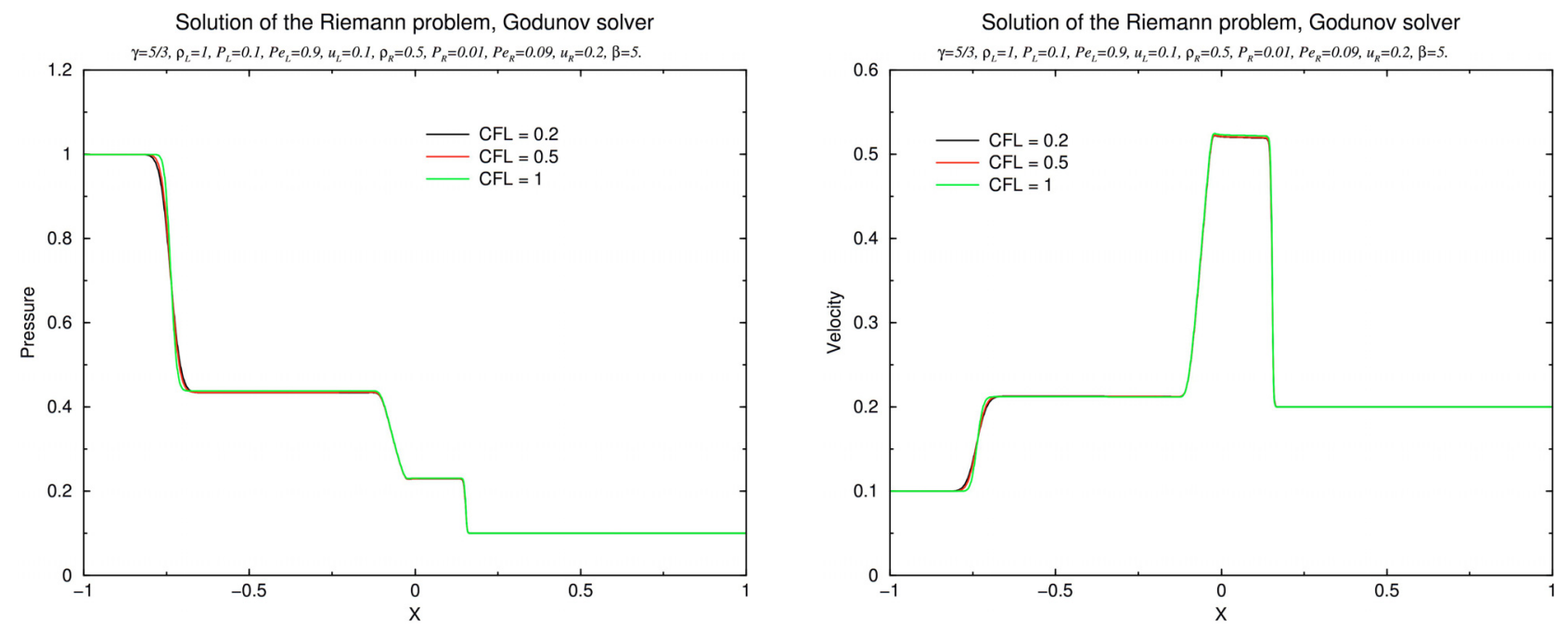

Figure 8.1. Godunov solver for different CFL's (pressure and velocity).

where, following (8.13),

$$
C_{j}^{n}==\gamma \frac{P_{j-1 / 2}^{n}+P_{j+1 / 2}^{n}}{\tau_{j-1 / 2}^{n}+\tau_{j+1 / 2}^{n}}, \quad \kappa_{e, j}^{n}=\gamma \frac{p_{e, j-1 / 2}^{n}+p_{e, j+1 / 2}^{n}}{s_{e, j-1 / 2}^{n}+s_{e, j+1 / 2}^{n}}
$$

Observe that (8.15) and (8.16) give two distinct values $v_{0 \pm}^{n}$ and $P_{0 \pm}^{n}$ for $v_{0}^{n}$ and $P_{0}^{n}$ respectively.

(i) The Lagrangian step. We then compute for $j \in \mathbb{Z}$

$$
\left\{\begin{array}{l}
x_{j}^{*}=x_{j}+\Delta t v_{j}^{n}, \\
\rho_{j+1 / 2}^{*}=\frac{\Delta m_{j+1 / 2}^{n}}{x_{j+1}^{*}-x_{j}^{*}}, \quad \Delta m_{j+1 / 2}^{n}=\Delta x \rho_{j+1 / 2}^{n}, \\
v_{j+1 / 2}^{*}=v_{j+1 / 2}^{n}-\frac{\Delta t}{\Delta m_{j+1 / 2}^{n}}\left(P_{j+1}^{n}-P_{j}^{n}\right), \\
s_{e, j}^{*}=\left\{\begin{array}{l}
s_{e, j+1 / 2}^{n}+\frac{\beta \Delta t}{\Delta m_{j+1 / 2}^{n}}\left(s_{e, j+1}^{n}-s_{e, j}^{n}\right), \quad j<0, \\
s_{e, j+1 / 2}^{n}, \quad j \geq 0,
\end{array}\right. \\
s_{j+1 / 2}^{*}=s_{j+1 / 2}^{n} .
\end{array}\right.
$$

Since $v_{0}^{n}$ and $P_{0}^{n}$ are not single-valued, $\rho_{ \pm 1 / 2}^{*}$ and $v_{ \pm 1 / 2}^{*}$ are not well defined. In fact, for $j=-1,0$, the second and third equations (8.18) have to be understood in the following sense

$$
\left\{\begin{array}{l}
\rho_{-1 / 2}^{*}=\frac{\Delta m_{-1 / 2}^{n}}{\Delta t\left(v_{0-}^{n}-v_{-1}^{n}\right)}, \rho_{1 / 2}^{*}=\frac{\Delta m_{1 / 2}^{n}}{\Delta t\left(v_{1}^{n}-v_{0+}^{n}\right)}, \\
v_{-1 / 2}^{*}=v_{-1 / 2}^{n}-\frac{\Delta t}{\Delta m_{-1 / 2}^{n}}\left(P_{0-}^{n}-P_{-1}^{n}\right), v_{1 / 2}^{*}=v_{1 / 2}^{n}-\frac{\Delta t}{\Delta m_{1 / 2}^{n}}\left(P_{1}^{n}-P_{0+}^{n}\right) .
\end{array}\right.
$$


(ii) The projection step. The cell quantities at time $t_{n+1}$ are defined as

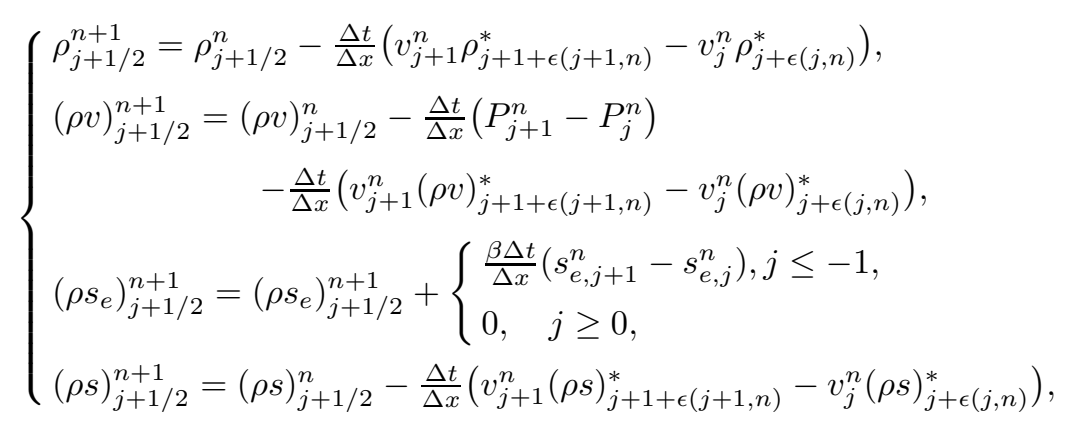

where

$$
\epsilon(j, n)=\left\{\begin{array}{l}
-1 / 2 \quad \text { if } v_{j}^{n}>0 \\
1 / 2 \quad \text { if } v_{j}^{n}<0 .
\end{array}\right.
$$

Again, for $j=-1$, the pair $\left(v_{0}^{n}, P_{0}^{n}\right)$ has to be understood as $\left(v_{0-}^{n}, P_{0-}^{n}\right)$, while for $j=1,\left(v_{0}^{n}, P_{0}^{n}\right)$ means $\left(v_{0+}^{n}, P_{0+}^{n}\right)$. We refer to [16] for details concerning the Lagrange-projection method. Let us notice that, at the difference with the HLLE method, all the components of the numerical flux of the Lagrange-projection method are discontinuous at $j=0$.

We now present some numerical illustrations concerning the solution of the coupled Riemann problem (7.10). We have used the following Riemann data.

\begin{tabular}{|c|c|c|c|c|c|}
\hline $\mathbf{u}$ & $\rho$ & $v$ & $v_{e}$ & $p$ & $p_{e}$ \\
\hline$L_{1}$ & 1 & 0.1 & -4.9 & 0.1 & 0.9 \\
\hline$L_{2}$ & 1 & 1 & -4 & 0.1 & 0.9 \\
\hline$R_{1}$ & 0.5 & 0.2 & 0.2 & 0.01 & 0.09 \\
\hline$R_{2}$ & 0.5 & 2 & 2 & 0.01 & 0.09 \\
\hline$R_{3}$ & 1 & 2 & 2 & 0.01 & 0.09 \\
\hline
\end{tabular}

We have chosen here $\beta=5$ (recall that $v_{e, L}=v_{L}-\beta / \rho_{L}, v_{e, R}=v_{R}$ ). Note that $P_{L}=1, P_{R}=0.1$. In order to study the dependence of the numerical solution on the data, we have chosen two different values for both the density $\rho_{R}$, the velocity $v$ and also for adiabatic exponent $\gamma$.

Example 8.1. The computations are done with $\mathbf{u}_{L_{1}}, \mathbf{u}_{R_{1}}, \gamma=5 / 3$. This set of values satisfies (7.9) and provides a $2-L$ wave $(v-c<0)$ at the interface. The results are given at time $t=0.15$.

The results (computations done with 2000 mesh points) do not seem to depend on the CFL or the dependence is unsignificant (see Fig. 8.1). On this test, the solution given by the HLLE scheme does not either depend on the CFL, and coincides with Godunov's solution which corresponds to $s_{e}^{*}=1,3 s_{e, R}$ (the computations in Fig. 8.2 are done at CFL 0.5 with 10000 mesh points).

Example 8.2. The computations are done with $\mathbf{u}_{L_{2}}, \mathbf{u}_{R_{2}}, \gamma=5 / 3$. This set of values also satisfies (7.9) but provides a $1-R$ wave $(v-c>0)$ at the interface ( $c f$. Rem. 7$)$. We show that the numerical solution depends only slightly on the numerical scheme. In Figure 8.3, we compare the solution for three first order schemes namely, the Godunov, HLLE solvers and a Lagrange-projection solver (with the Roe solver in the Lagrangian step). The CFL is 0.2 and we have taken 10000 mesh points, the results are given at time $t=0.2$.

In Figure 8.4, we compare the HLLE scheme with its 2nd order MUSCL extension. The common converged solution is a solution of the coupled Riemann problem corresponding to $s_{e}^{*}=1,35 s_{e, R}$ (see Fig. 8.4, right, where we compare to this analytical solution).

In Figure 8.5 we compare the solution for the Godunov solver corresponding to different CFL's on 2000 mesh points. 

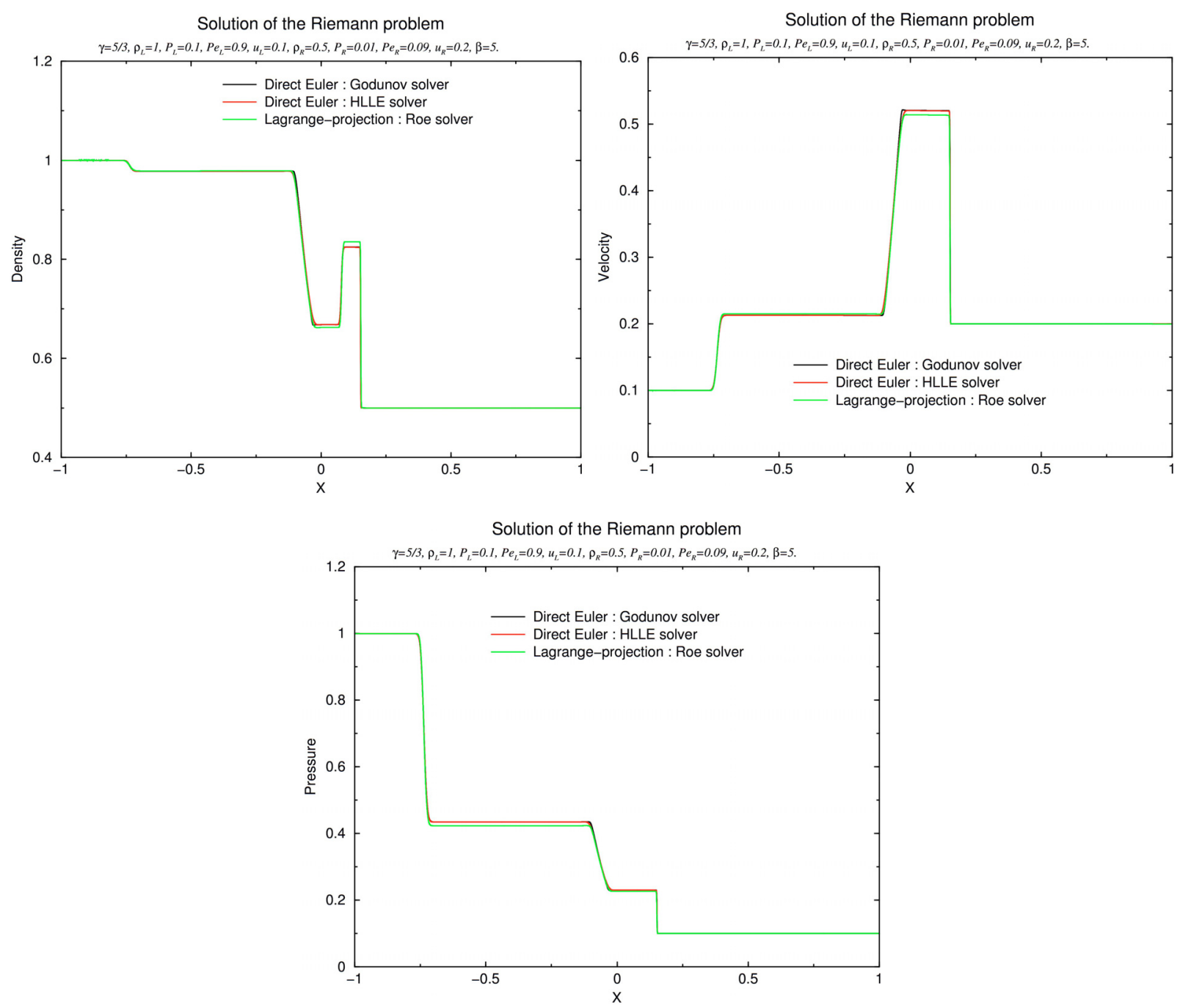

Figure 8.2. Comparison of different schemes.

For Godunov's scheme the numerical solutions do not seem to depend on the CFL (or the dependence is unsignificant). For the HLLE solver on the same test however, we have noticed a slight dependence of the level of the constant states on the CFL.

Note also that the converged solution provided by Godunov's scheme is a solution of the coupled Riemann problem corresponding to a slightly different fitted coefficient $s_{e}^{*}=1,34 s_{e, R}$ (this is confirmed by plotting the error $\left.\Sigma\left|\rho_{i}-\rho\left(x_{i}\right)\right| \Delta x\right)$. We now give the error between the schemes $\left(\Sigma\left|\rho_{i}^{a}-\rho_{i}^{b}\right| \Delta x\right.$, with obvious notations for $a, b)$ which tends to prove that the numerical converged solutions given by the Roe and HLLE schemes coincide and differ slightly from Godunov's (Fig. 8.7).

Example 8.3. We show here the influence of various $\gamma$ 's. The computations in Figure 8.8 are done with $\mathbf{u}_{L_{2}}, \mathbf{u}_{R_{3}}$ left $\gamma=7 / 5$, right $\gamma=5 / 3$.

We observe that $s_{e}^{*}$ indeed depends on $\gamma: s_{e}^{*}=2 s_{e, R}$ left, $s_{e}^{*}=1,725 s_{e, R}$ right, and moreover, comparing with Example 8.2 , we see that $s_{e}^{*}$ depends on the initial density. 

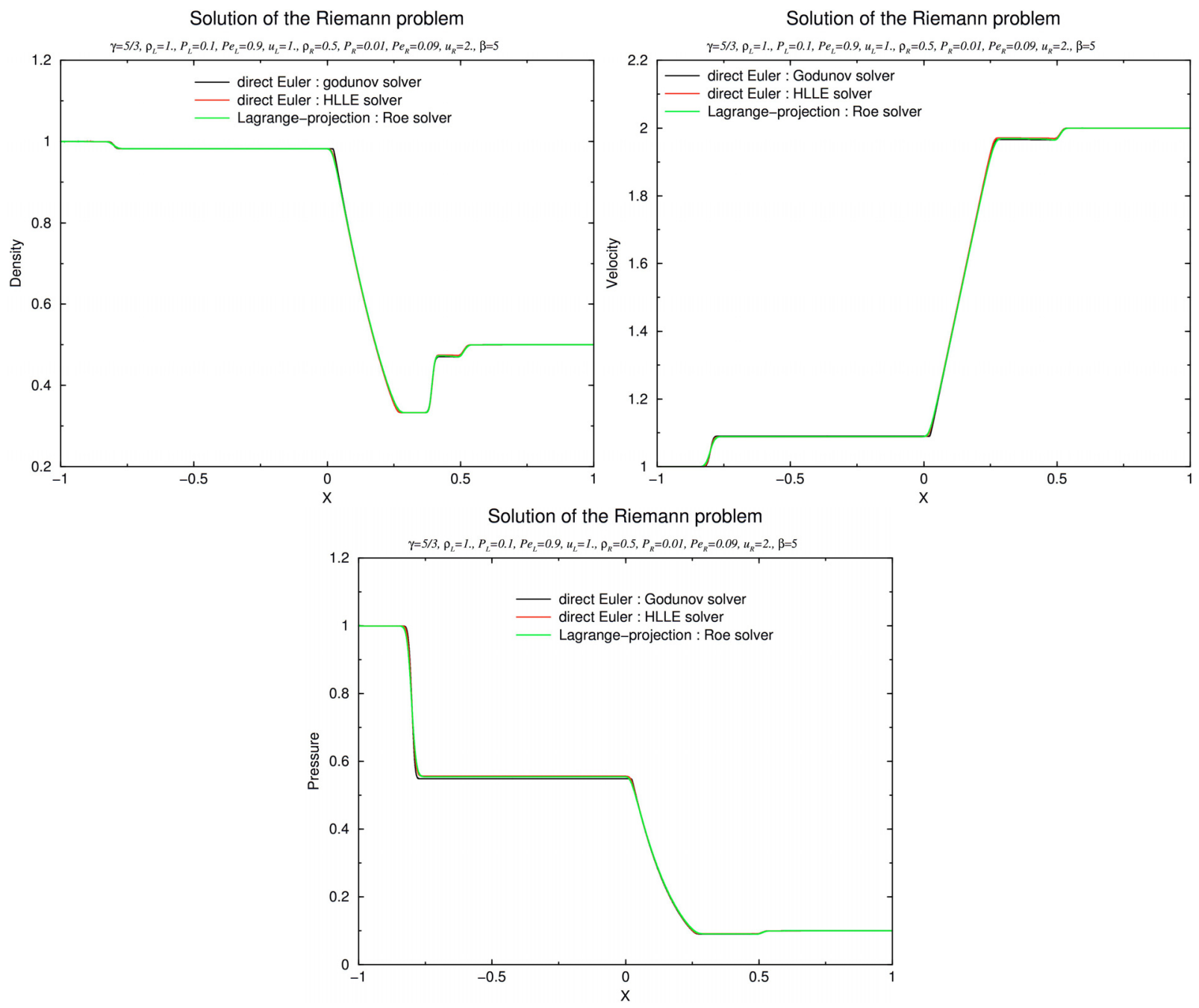

FiguRE 8.3. Solution of the Riemann problem (density, velocity, pressure) with different 1 st order schemes.

We have also considered the effect of regularizing the initial data. We start from a Riemann problem between $\mathbf{u}_{L_{2}}$ and $\mathbf{u}_{R_{3}}$, where the initial density is continuous $\left(\rho_{R}=\rho_{L}=1, \gamma=5 / 3\right)$ and the initial velocity and pressure are discontinuous. We regularize these velocity and pressure using a cubic spline, depending on a parameter $\varepsilon$ tending to 0 (Fig. 8.9).

Comparing with the results of Example 8.3 (see Fig. 8.8, right), we observe that the solution is indeed different and corresponds to $s_{e}^{*}=2.8 s_{e, R}$.

We have thus shown that in the case of discontinuous initial data all our numerical schemes converge towards (almost) the same solution of the coupled Riemann solution that we cannot characterize simply. Moreover, this solution is not stable with respect to a regularizing process. In the scalar case however we have seen ([17], corollary of Thm. 7) that, when the initial data $u_{0}$ is continuous, the sequence of discrete solutions converges to the unique solution of the coupled problem corresponding to $u_{0}(0+)=u_{0}(0-)=u_{0}(0)$. Numerical experiments in the case of the coupled plasma problem with continuous initial data have lead indeed to realistic results for physically complex problems (cf. [26]). 

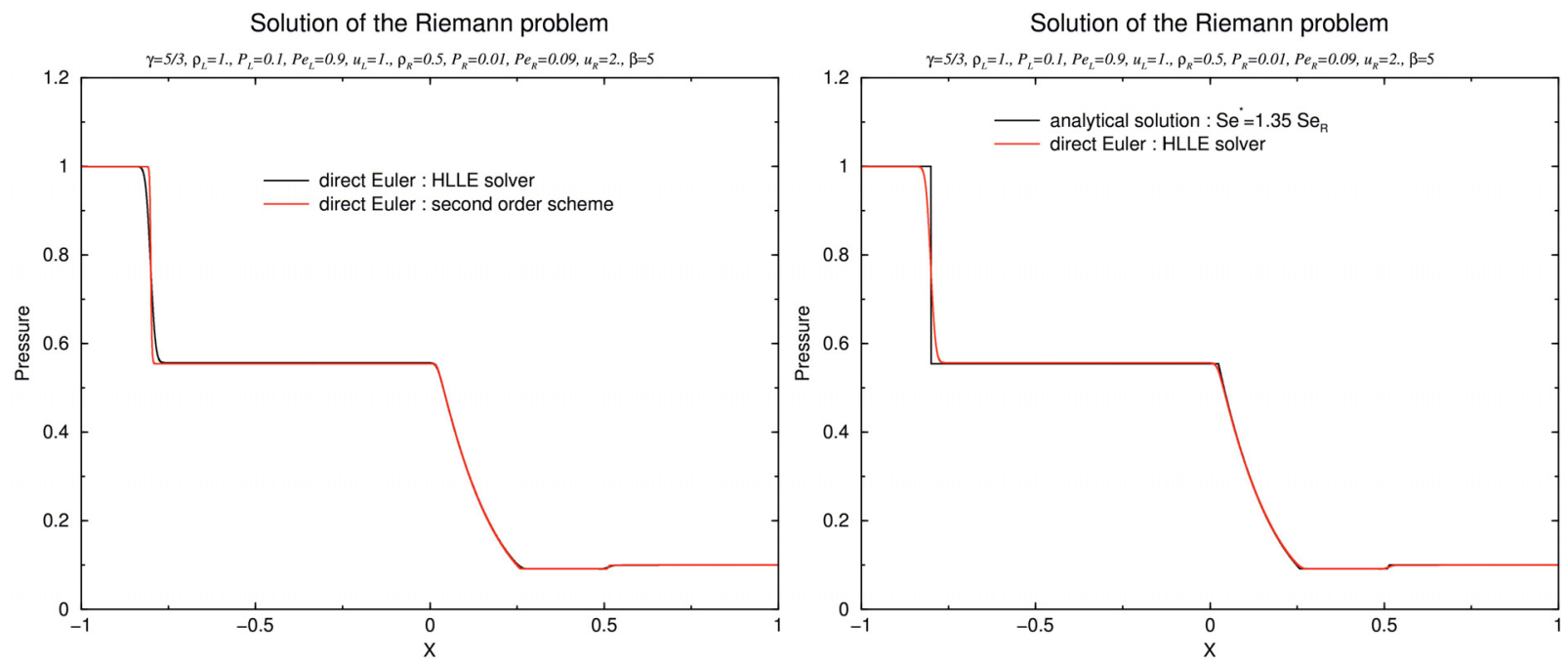

FiguRE 8.4. Solution of the Riemann problem (pressure): left with HLLE schemes (1st order and 2nd order extension) - right HLLE and analytical.
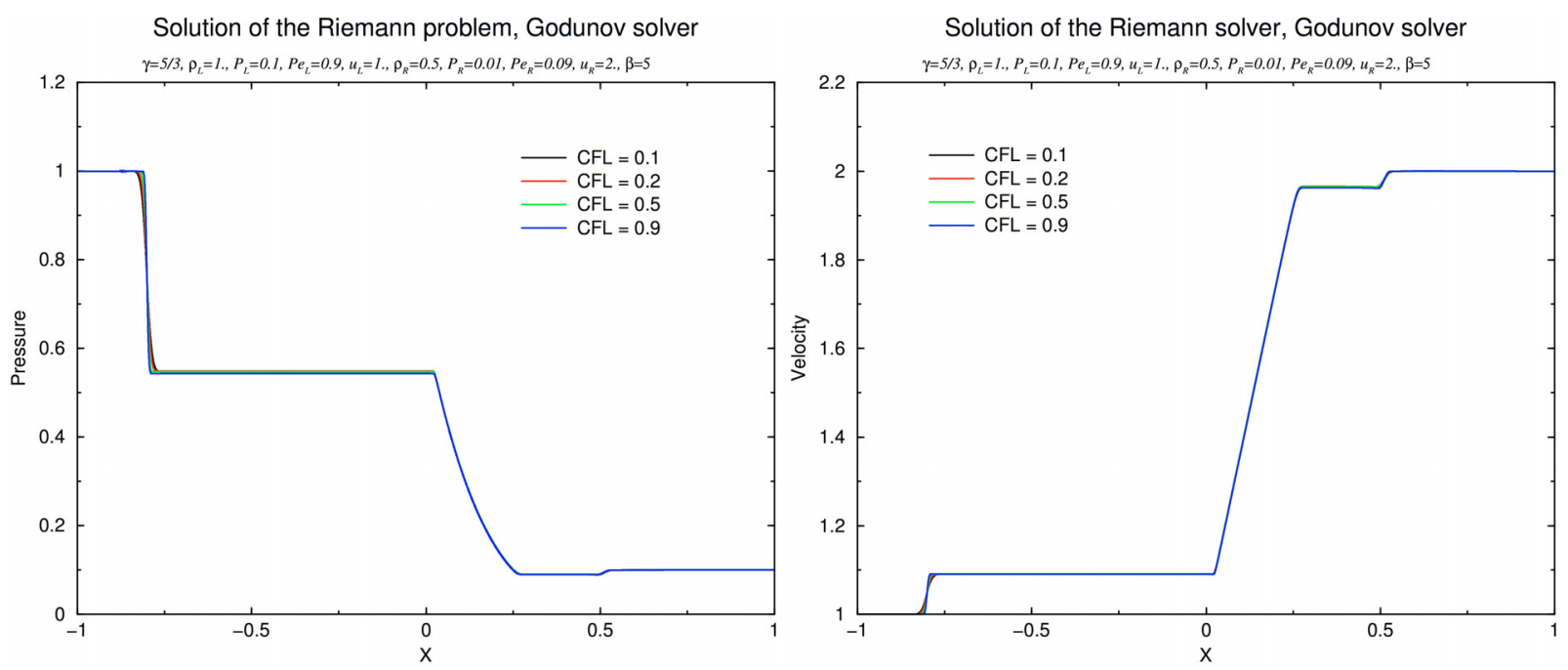

FIGURE 8.5. Godunov solver for different CFL's (pressure and velocity).

\section{Conclusion}

In this paper, we have continued the study of our method of coupling systems of conservation laws of the same dimension at an interface. This method is based on a weak continuity constraint for the solution at the interface. In the case of linear systems with constant coefficients, we have been able to explicit the coupling conditions and show that the solution of the coupled system may not be unique in some cases, depending on the geometry of characteristics at the interface. We have given extensions of the coupling method to some nonlinear cases of practical interest. In the particular case of the coupled plasma problem, we have checked that the numerical method works satisfactorily, even in cases of nonuniqueness of the solution. On the one hand, the numerical solution depends only slightly on the numerical scheme and the wave velocities do not seem to 

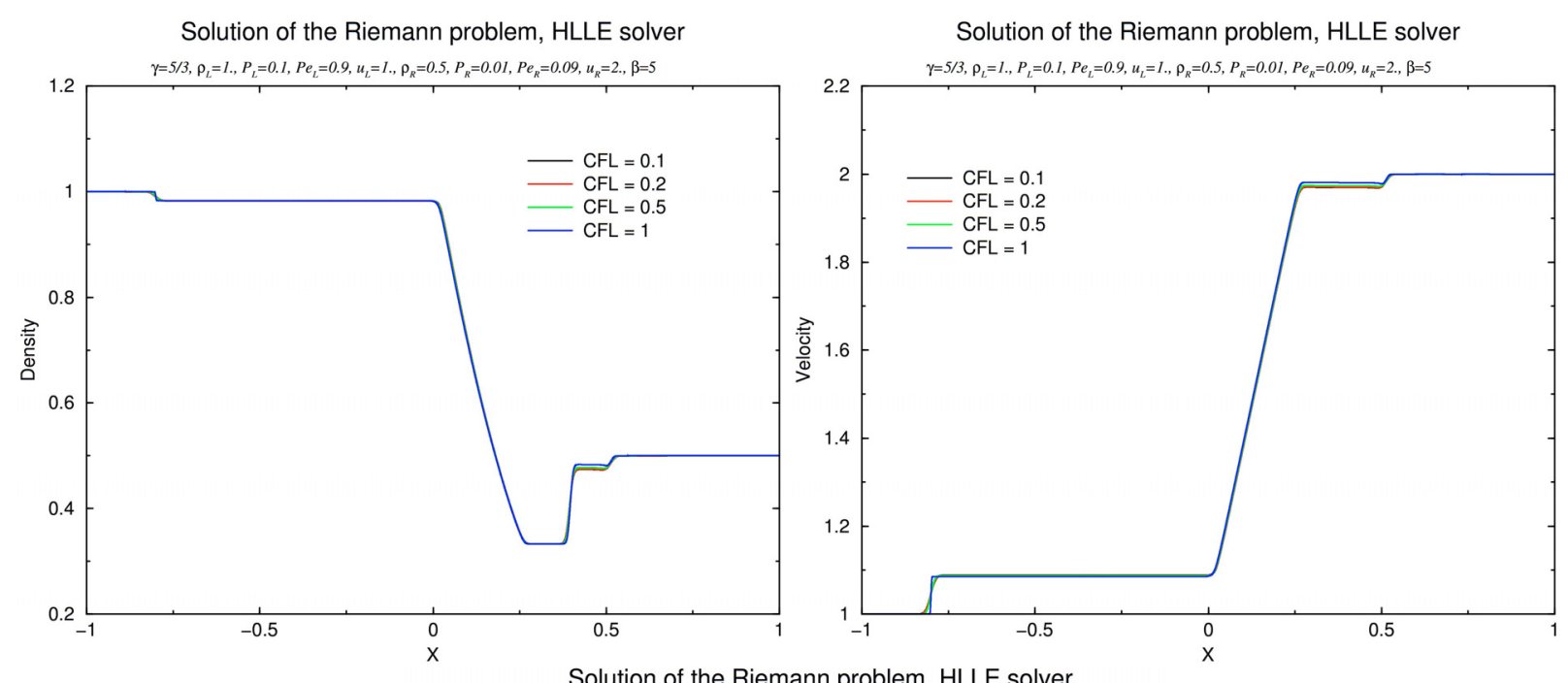

Solution of the Riemann problem, HLLE solver

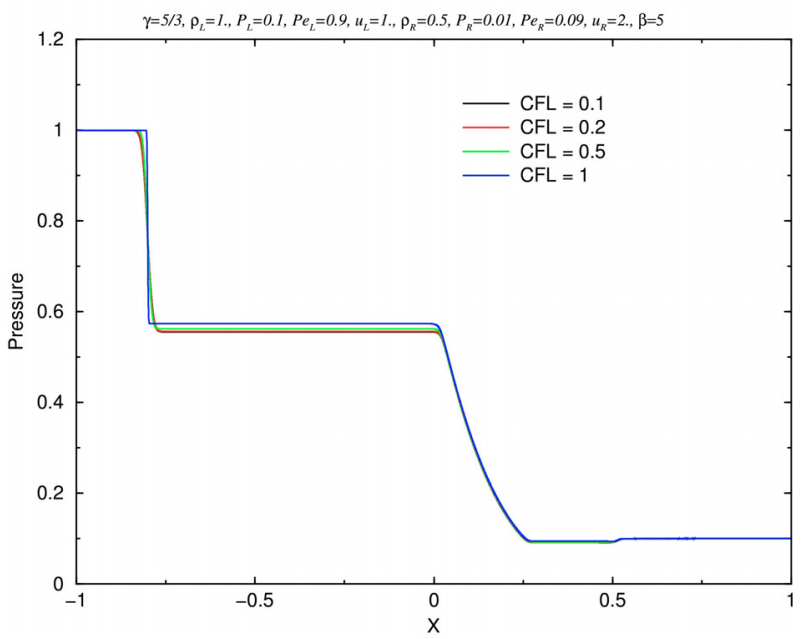

Figure 8.6. HLLE solver for different CFL's.

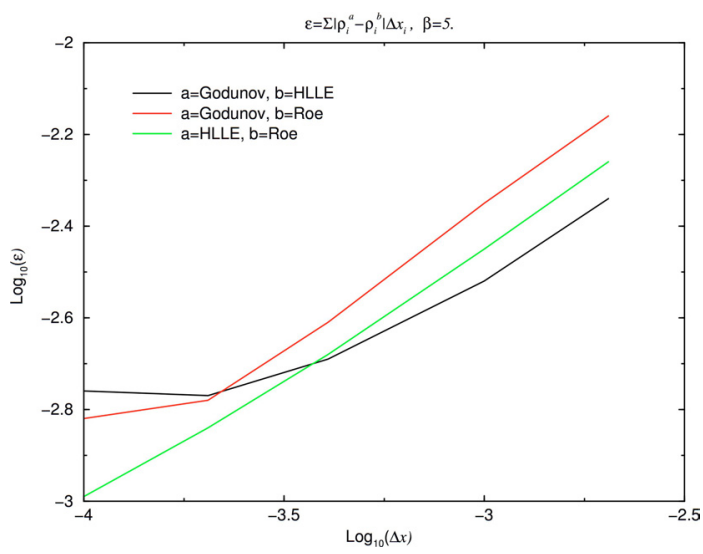

Figure 8.7. Plot of the error between two schemes. 

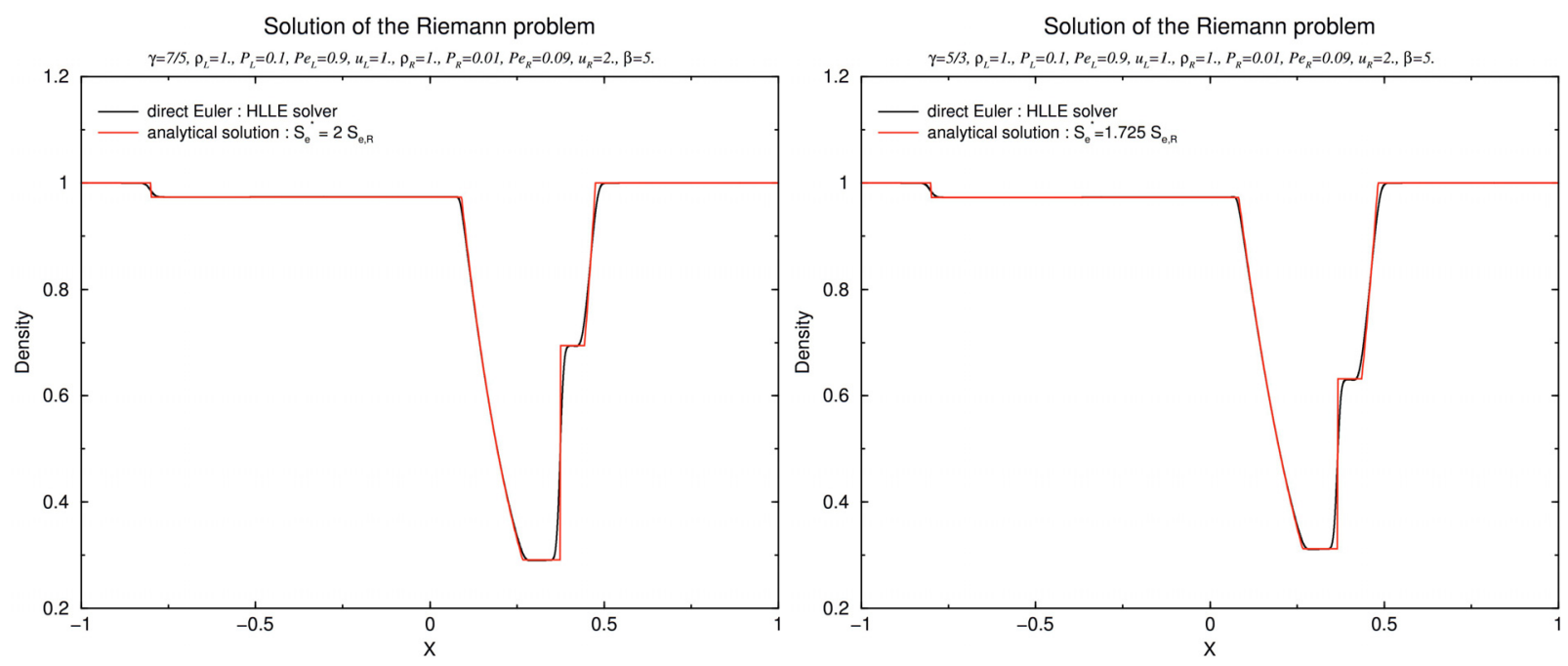

Figure 8.8. Solution of the Riemann problem for two different $\gamma$ 's.
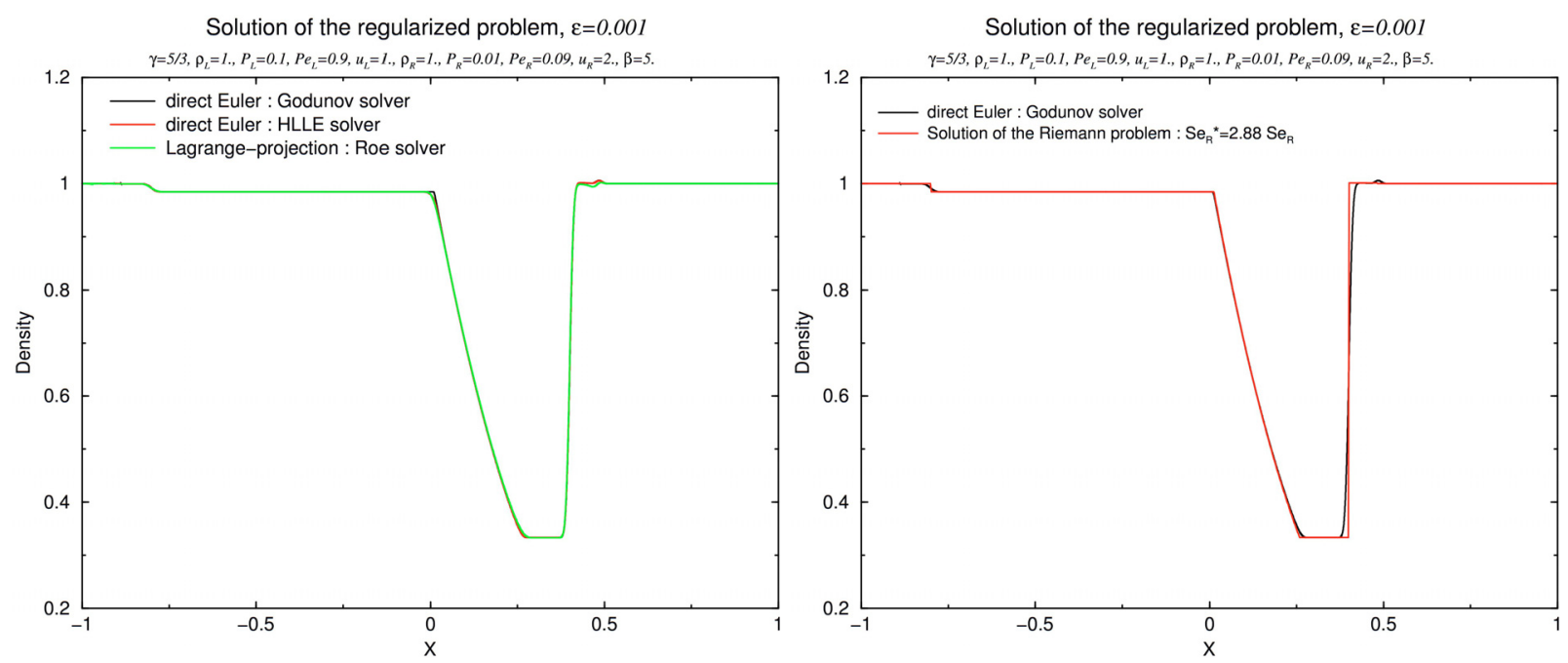

FIGURE 8.9. Solution of the regularized Riemann problem.

depend on the scheme. On the other hand, resonance does not appear in the situation where an eigenvalue jumps over another one at the interface.

It remains to understand the behavior of numerical schemes but we cannot expect to obtain easily theoretical results. Indeed, at the difference with the scalar case, there are very few theoretical results concerning the numerical approximation of the boundary value problem for systems (see however [15]). In particular, in the case of nonuniqueness of the solution of the coupled problem, the numerical method selects a "natural" one but we have no precise mathematical criterion for predicting or characterizing this natural solution though numerical experiments on plasma fluid models are constructive.

The paper may me viewed as a first contribution to the study of the coupling of different first order hyperbolic models occurring in industrial problems. The next step in such a study consists in coupling gas dynamics systems with different equations of state, and comparing our approach with a flux coupling method. It is also possible to approach the numerical coupling via an interface model as suggested in Remark 1. Indeed, following 
and extending the ideas of Section 4, the program can be completed when the two systems are written in Lagrangian coordinates. The problem is more difficult in Eulerian coordinates since the signs of the eigenvalues of the Jacobian matrix may change through the interface so that the geometry of characteristics at the interface is not fixed. We refer to [6] for a fairly complete analysis in Lagrangian coordinates and a still partial analysis in Eulerian coordinates. We have tested the numerical coupling of two such systems at a fixed interface. Note that we have not considered in this paper the case of a moving interface, for instance a material interface between two fluids in Eulerian coordinates. This creates other numerical difficulties which were taken up in [1].

A second typical problem consists in coupling an hyperbolic model with a stiff relaxation source term and the corresponding relaxed model. Such question is frequently met in the context of thermohydraulics. Although the two systems are of different dimensions, coupling conditions are easy to formulate together with corresponding numerical methods, but the problem is still essentially open.

Acknowledgements. The authors would like to thank warmly the referees for their many fruitful suggestions and interesting comments.

\section{Appendix A. Proof of Lemma 8 and COMplements}

We begin with the proof of Lemma 8 . We first observe that the function $f(. ; a)$ is strictly decreasing from $+\infty$ to $(\gamma+1)\left(\frac{a}{\gamma}\right)^{\frac{\gamma}{\gamma+1}}$ in the interval $\left(0,\left(\frac{a}{\gamma}\right)^{\frac{\gamma}{\gamma+1}}\right]$ and strictly increasing from $(\gamma+1)\left(\frac{a}{\gamma}\right)^{\frac{\gamma}{\gamma+1}}$ to $+\infty$ in the interval $\left[\left(\frac{a}{\gamma}\right)^{\frac{\gamma}{\gamma+1}},+\infty\right)$.

Hence, for a given $a>0$, the equation

$$
f(\xi ; a)=\alpha
$$

has at least one solution $\xi$ if and only if

$$
\alpha \geq(\gamma+1)\left(\frac{a}{\gamma}\right)^{\frac{\gamma}{\gamma+1}} .
$$

It has exactly one solution if $\alpha=(\gamma+1)\left(\frac{a}{\gamma}\right)^{\frac{\gamma}{\gamma+1}}$, and two solutions if $\alpha>(\gamma+1)\left(\frac{a}{\gamma}\right)^{\frac{\gamma}{\gamma+1}}$.

Consider next equation (6.25) where $b>0$ is a parameter. Assume first $b>a$. Then (6.25) has at least one solution if and only if

Since, for $b>a$, the equation

$$
f(\xi ; a) \geq(\gamma+1)\left(\frac{b}{\gamma}\right)^{\frac{\gamma}{\gamma+1}}
$$

has two solutions

$$
f(\xi ; a)=(\gamma+1)\left(\frac{b}{\gamma}\right)^{\frac{\gamma}{\gamma+1}}
$$

$$
\xi_{1}(b)<\left(\frac{b}{\gamma}\right)^{\frac{\gamma}{\gamma+1}}<\xi_{2}(b)
$$

we find that, for $\xi<\xi_{1}(b)$ or $\xi>\xi_{2}(b)$, equation (6.25) has exactly two solutions $\eta_{1}(\xi ; b)$ and $\eta_{2}(\xi ; b)$ which satisfy

$$
\left\{\begin{array}{l}
\eta_{1}(\xi ; b)<\left(\frac{b}{\gamma}\right)^{\frac{\gamma}{\gamma+1}}<\eta_{2}(\xi ; b) \\
\eta_{1}(\xi ; b)>\xi \text { for } \xi<\xi_{1}(b), \eta_{2}(\xi ; b)<\xi \text { for } \xi>\xi_{2}(b) .
\end{array}\right.
$$

As $b$ varies, we obtain two branches of solutions $\eta_{i}(\xi ; \cdot): b \rightarrow \eta_{i}(\xi ; b), i=1,2$. Setting

$$
b_{\max }(\xi)=\gamma\left(\frac{f(\xi ; a)}{\gamma+1}\right)^{\frac{\gamma+1}{\gamma}},
$$




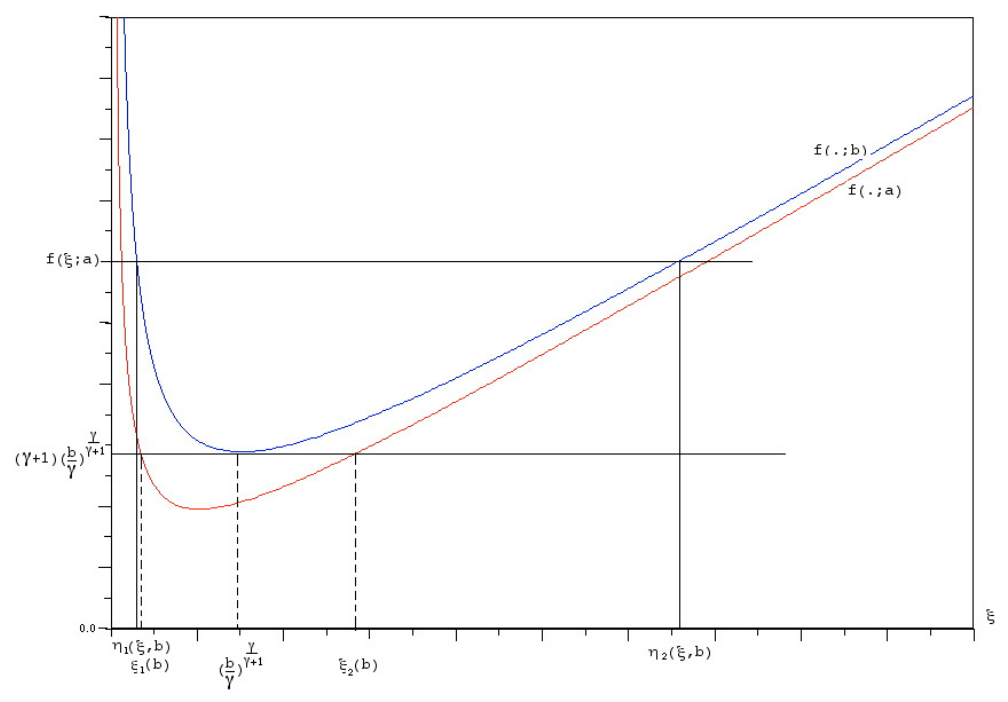

FiguRE A.1.

each branch $\eta_{i}(x ; \cdot)$ is defined in $\left[a, b_{m} a x(\xi)\right]$. A simple computation gives

$$
\frac{\partial \eta_{i}}{\partial b}=-\frac{\eta_{i}}{\eta_{i}^{\frac{\gamma+1}{\gamma}}-\frac{b}{\gamma}} .
$$

Since

$$
\frac{\eta}{\eta^{\frac{\gamma+1}{\gamma}}-\frac{b}{\gamma}}\left\{\begin{array}{l}
<0, \text { if } \eta<\left(\frac{b}{\gamma}\right)^{\frac{\gamma}{\gamma+1}} \\
>0, \text { if } \eta>\left(\frac{b}{\gamma}\right)^{\frac{\gamma}{\gamma+1}}
\end{array}\right.
$$

we get

$$
\frac{\partial \eta_{1}}{\partial b}(\xi ; b)>0, \frac{\partial \eta_{2}}{\partial b}<0,\left|\frac{\partial \eta_{i}}{\partial b}\left(\xi ; b_{\max }\right)\right|=+\infty,
$$

so that we obtain the following graph depicted on Figure A.2 for instance in the case $\xi<\left(\frac{b}{\gamma}\right)^{\frac{\gamma}{\gamma+1}}$.

Note that for $\xi<\frac{f(\xi ; a)}{\gamma+1}$, i.e. for $\xi<\frac{a}{\gamma} \frac{\gamma}{\gamma+1}$, we have $\eta_{1}(\xi ; a)=\xi$, while for $\xi>\frac{f(\xi ; a)}{\gamma+1}$, or for $\xi>\frac{a}{\gamma} \frac{\gamma}{\gamma+1}$, we obtain $\eta_{2}(\xi ; a)=\xi$. Hence, if we require for the solution of (6.25) to satisfy the continuity property

$$
\eta(\xi ; b) \rightarrow \xi \text { as } b \rightarrow a,
$$

we obtain

$$
\eta(\xi ; b)= \begin{cases}\eta_{1}(\xi ; b), \quad \xi \leq\left(\frac{a}{\gamma}\right)^{\frac{\gamma}{\gamma+1}} \\ \eta_{2}(\xi ; b), \quad \xi \geq\left(\frac{a}{\gamma}\right)^{\frac{\gamma}{\gamma+1}} .\end{cases}
$$

Consider next the case $0<a<b$ (the case $b=a$ being obvious). Then clearly equation (6.25) has always two solutions $\eta_{i}(. ; b), i=1,2$ and the above analysis still applies. This ends the proof of Lemma 8.

We will need in Appendix B the following estimates for the derivative $\frac{\partial \eta}{\partial \xi}$ of the function $\eta$ defined by $(A .2)$

$$
0 \leq \frac{\partial \eta}{\partial \xi}(\xi ; b) \leq 1
$$




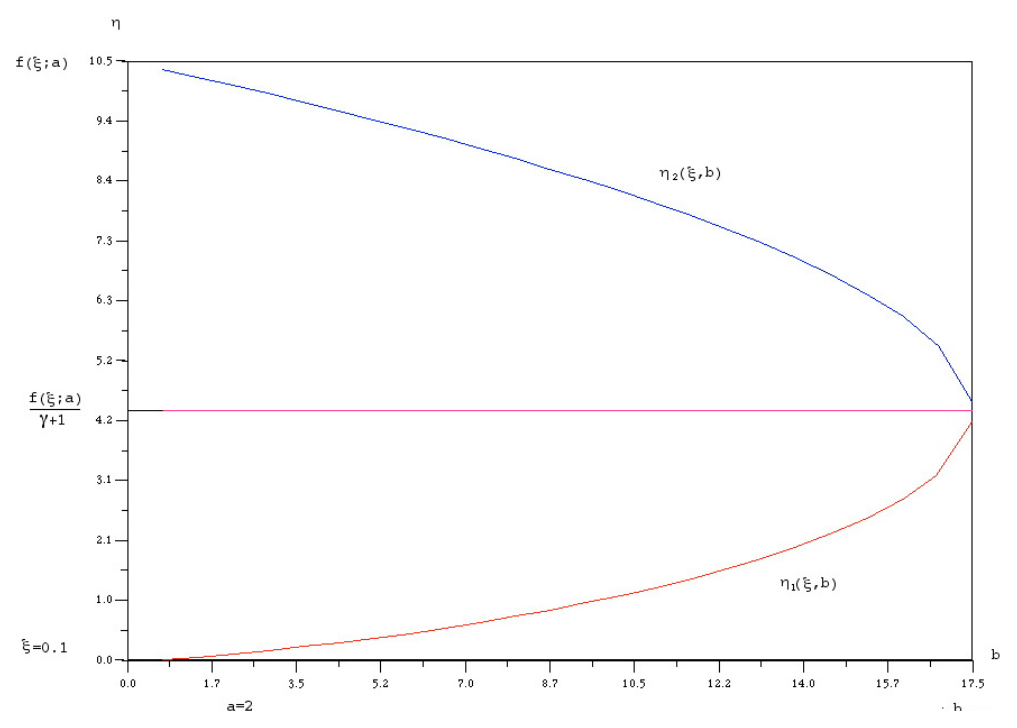

Figure A.2.

Assume first $\xi<\left(\frac{a}{\gamma}\right)^{\frac{\gamma}{\gamma+1}}$ so that

$$
\eta(\xi, b)=\eta_{1}(\xi ; b)
$$

Since

$$
f\left(\eta_{1} ; b\right)=f(\xi ; a) \Longleftrightarrow 1+a \xi^{-\frac{\gamma}{\gamma+1}}=\frac{\eta_{1}}{\xi}\left(1+b \eta_{1}^{-\frac{\gamma}{\gamma+1}}\right)
$$

and

$$
\eta_{1}<\xi
$$

we have

$$
a \xi^{-\frac{\gamma+1}{\gamma}}<b \eta_{1}^{-\frac{\gamma+1}{\gamma}}
$$

and therefore

Hence, we obtain

$$
\frac{\partial f}{\partial \xi}\left(\eta_{1} ; b\right)=1-\frac{b}{\gamma} \eta_{1}^{-\frac{\gamma+1}{\gamma}}<1-\frac{a}{\gamma} \xi^{-\frac{\gamma+1}{\gamma}}=\frac{\partial f}{\partial \xi}(\xi ; a)
$$

which yields

$$
\frac{\partial f}{\partial \xi}\left(\eta_{1} ; b\right)<\frac{\partial f}{\partial \xi}(\xi ; a)<0
$$

$$
0<\frac{\partial \eta_{1}}{\partial \xi}(\xi ; b)=\frac{\frac{\partial f}{\partial \xi}(\xi ; a)}{\frac{\partial f}{\partial \xi}\left(\eta_{1}(\xi ; b) ; b\right)}<1
$$

This proves $(A .3)$ for $\xi<\left(\frac{a}{\gamma}\right)^{\frac{\gamma}{\gamma+1}}$.

On the other hand, for $\xi>\left(\frac{a}{\gamma}\right)^{\frac{\gamma}{\gamma+1}}$, we find

$$
\frac{\partial f}{\partial \xi}\left(\eta_{2} ; b\right)>\frac{\partial f}{\partial \xi}(\xi ; a)>0
$$

which again implies (A.3). Finally, the bounds of $(A .3)$ are reached for $\xi=\left(\frac{a}{\gamma}\right)^{\frac{\gamma}{\gamma+1}}$. 


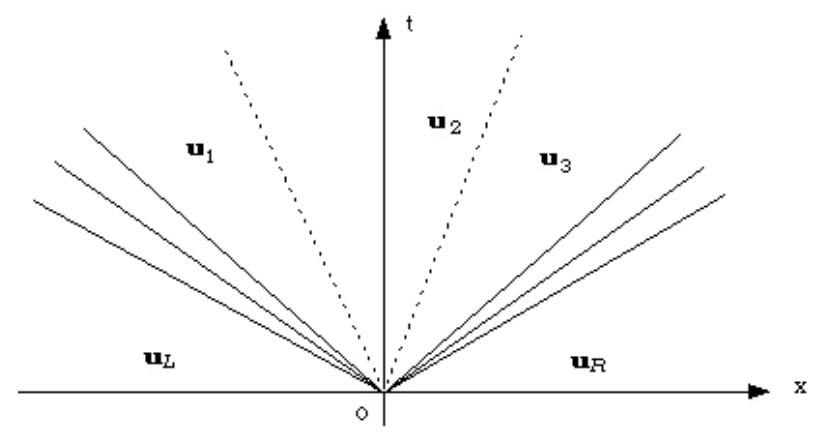

Figure B.1.

\section{Appendix B. The Riemann problem for the Coupled Plasma systems in the CASE}

$$
v_{L}-c_{L}<v_{e, L}<0 \text {. }
$$

The purpose of this Appendix is to solve the coupled problem (7.10) when the state $\mathbf{u}_{L}$ satisfies

$$
v_{L}-c_{L}<v_{e, L}<0 .
$$

We begin by considering the Riemann problem (6.5), (6.6) with the closure relation (6.3). When the condition (B.1) holds, the solution of the Riemann problem is then of the form depicted in Figure B.1.

The relations between the states $\mathbf{u}_{L}, \mathbf{u}_{1}, \mathbf{u}_{2}, \mathbf{u}_{3}$, and $\mathbf{u}_{R}$ read

$$
\begin{gathered}
\left\{\begin{array}{l}
v_{1}=v_{L}-\Phi_{L}\left(P_{1}\right), \\
s_{1}=s_{L}, \\
s_{e, 1}=s_{e, L},
\end{array}\right. \\
\left\{\begin{array}{l}
v_{e, 2}=v_{e, 1}, \\
\alpha\left(v_{2}-v_{1}\right)+P_{2}-P_{1}=0, \\
s_{2}=s_{1},
\end{array}\right. \\
\left\{\begin{array}{l}
v_{3}=v_{2}, \\
P_{3}=P_{2}, \\
s_{e, 3}=s_{e, 2},
\end{array}\right.
\end{gathered}
$$

and

Note that

$$
\left\{\begin{array}{l}
v_{3}=v_{R}+\Phi_{R}\left(P_{3}\right), \\
s_{3}=s_{R} \\
s_{e, 3}=s_{e, R} .
\end{array}\right.
$$

We set

$$
\left\{\begin{array}{l}
s_{1}=s_{2}=s_{L}, s_{3}=s_{R}, \\
s_{e, 1}=s_{e, L}, s_{e, 2}=s_{e, 3}=s_{e, R} .
\end{array}\right.
$$

$$
v_{*}=v_{2}=v_{3}, P_{*}=P_{2}=P_{3} .
$$

Then, for solving the Riemann problem, a first step consists in forming a system of two equations in $\left(v_{*}, P_{*}\right)$. On the one hand, the first equation (B.4) reads

$$
v_{*}=v_{R}+\Phi_{R}\left(P_{*}\right) .
$$


On the other hand, for establishing a second equation in $\left(v_{*}, P_{*}\right)$, we start from the first equation (B.3) which yields

where

$$
v_{*}-\alpha\left(\frac{K_{2}}{P_{*}}\right)^{\frac{1}{\gamma}}=v_{1}-\alpha\left(\frac{K_{1}}{P_{1}}\right)^{\frac{1}{\gamma}}
$$

In addition, the second equation $(B .2)$ reads

$$
K_{1}=s_{L}^{\gamma}+s_{e, L}^{\gamma}=K_{L}, K_{2}=s_{L}^{\gamma}+s_{e, R}^{\gamma} .
$$

$$
\alpha\left(v_{*}-v_{1}\right)+P_{*}-P_{1}=0 .
$$

Combining equations $(B .6)$ and (B.7), we obtain

$$
P_{1}+\alpha^{2}\left(\frac{K_{1}}{P_{1}}\right)^{\frac{1}{\gamma}}=P_{*}+\alpha^{2}\left(\frac{K_{2}}{P_{*}}\right)^{\frac{1}{\gamma}}
$$

The above equation enables us to determine $P_{1}$ from $P_{*}$ at least under suitable conditions. Indeed, using Lemma 8, the following holds:

(i) for $K_{2} \geq K_{1}$ or $s_{e, R} \geq s_{e, L}$, equation (B.8) has a unique admissible solution $P_{1}=P_{1}\left(P_{*}\right)$;

(ii) for $K_{2}<K_{1}$ or $s_{e, R}<s_{e, L}$, equation (B.8) has a unique admissible solution provided that

$$
P_{*}+\alpha^{2}\left(\frac{K_{2}}{P_{*}}\right)^{\frac{1}{\gamma}} \geq(\gamma+1)\left(\alpha^{2} \frac{K_{2}^{\frac{1}{\gamma}}}{\gamma}\right)^{\frac{\gamma}{\gamma+1}}
$$

which amounts to suppose $P_{*}$ small enough or large enough.

For the sake of simplicity, we assume here

$$
s_{e, R} \geq s_{e, L},
$$

so that the function $P_{*} \rightarrow P_{1}\left(P_{*}\right)$ is defined for $P_{*} \geq 0$. Using the inequality $(A .3)$, we obtain in addition

$$
0 \leq \frac{\mathrm{d} P}{\mathrm{~d} P_{*}} \leq 1
$$

Next, combining the first equation (B.2) with (B.7), we find

$$
v_{*}=v_{L}-\left(\Phi_{L}\left(P_{1}\right)+\frac{1}{\alpha}\left(P_{*}-P_{1}\right)\right) .
$$

Setting

the above equation reads

$$
\Psi_{L}\left(P_{*}\right)=\Phi_{L}\left(P_{1}\left(P_{*}\right)\right)+\frac{1}{\alpha}\left(P_{*}-P_{1}\left(P_{*}\right)\right)
$$

$$
v_{*}=v_{L}-\Psi_{L}\left(P_{*}\right)
$$

hence, the pair $\left(v_{*}, P_{*}\right)$ is solution of the system of equations $(B .5),(B .12)$.

Now, since by (B.10)

$$
\frac{\mathrm{d} \Psi_{L}}{\mathrm{~d} P_{*}}=\frac{\mathrm{d} \Phi_{L}}{\mathrm{~d} P_{1}} \frac{\mathrm{d} P_{1}}{\mathrm{~d} P_{*}}+\frac{1}{\alpha}\left(1-\frac{\mathrm{d} P_{1}}{\mathrm{~d} P_{*}} \geq \frac{\mathrm{d} \Phi_{L}}{\mathrm{~d} P_{1}} \frac{\mathrm{d} P_{1}}{\mathrm{~d} P_{*}} \geq 0,\right.
$$

the function $\Psi_{L}$ is monotonically increasing. As the function $\Phi_{R}$ is strictly increasing, the system $(B .5),(B .12)$ has a unique solution $\left(v_{*}, P_{*}\right)$ if and only if

$$
v_{L}-\Psi_{L}(0) \geq v_{R}+\Psi_{R}(0) .
$$




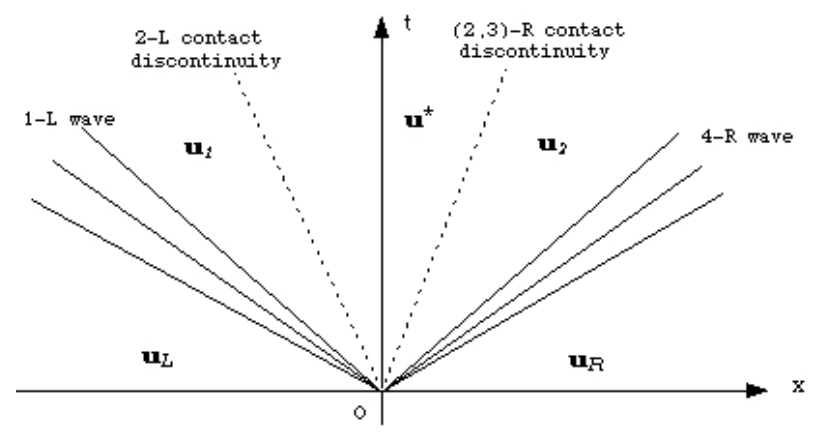

Figure B.2.

Since $P_{1}(0)=0$, we have $\Psi_{L}(0)=\Phi_{L}(0)$ and the above condition gives

$$
v_{L}-\Phi_{L}(0) \geq v_{R}+\Phi_{R}(0),
$$

which is nothing but the usual condition in gas dynamics for avoiding vacuum in the solution of the Riemann problem. If the condition (B.13) does not hold, we have to allow vacuum to appear in the solution of $(6.5)$, (6.6). In any case, under the hypotheses (B.1) and (B.9), the Riemann problem for (6.5), (6.6) has a unique self similar solution of the above form. The discussion of the case $s_{e, R}<s_{e, L}$ is analogous and left to the reader.

We pass to the coupling problem. We first notice that the analogue of Lemma 9 holds with the same proof. If we assume that the states $\mathbf{u}(0-, t)$ and $\mathbf{u}(0+, t)$ satisfy

$$
\left\{\begin{array}{l}
(v-c)(0-, t)<v_{e}(0-, t)=\left(v-\frac{\alpha}{\rho}\right)(0-, t)<0<(v+c)(0-, t) \\
(v-c)(0+, t)<0<v_{e}(0+, t)=v(0+, t)<(v+c)(0+, t)
\end{array}\right.
$$

the coupling conditions (2.6) are again equivalent to the continuity constraint (7.4). We next suppose that the two states $\mathbf{u}_{L}$ and $\mathbf{u}_{R}$ satisfy

$$
\left\{\begin{array}{l}
v_{L}-c_{L}<v_{e, L}<0<v_{L}<v_{L}+c_{L} \\
v_{R}-c_{R}<0<v_{e, R}=v_{R}<v_{R}+c_{R}
\end{array}\right.
$$

Then we look for a self-similar solution of (7.10) of the form

Hence we have

$$
\begin{gathered}
\left\{\begin{array}{l}
v_{1}=v_{L}-\Phi_{L}\left(P_{1}\right), \\
s_{1}=s_{L}, \\
s_{e, 1}=s_{e, L},
\end{array}\right. \\
\left\{\begin{array}{l}
v_{e}^{*}=v_{e, 1}, \\
\alpha\left(v^{*}-v_{1}\right)+P^{*}-P_{1}=0, \\
s^{*}=s_{1},
\end{array}\right. \\
\left\{\begin{array}{l}
v_{2}=v^{*}, \\
P_{2}=P^{*},
\end{array}\right.
\end{gathered}
$$

and

$$
\left\{\begin{array}{l}
v_{2}=v_{R}+\Phi_{R}\left(P_{2}\right) \\
s_{2}=s_{R} \\
s_{e, 2}=s_{e, R}
\end{array}\right.
$$

Then choosing arbitrarily $s_{e}^{*}$ and arguing as above, we obtain

$$
P_{1}+\alpha^{2}\left(\frac{K_{1}}{P_{1}}\right)^{\frac{1}{\gamma}}=P^{*}+\alpha^{2}\left(\frac{K^{*}}{P^{*}}\right)^{\frac{1}{\gamma}}
$$


with

$$
K_{1}=K_{L}=s_{L}^{\gamma}+s_{e, L}^{\gamma}, \quad K^{*}=s_{L}^{\gamma}+\left(s_{e}^{*}\right)^{\gamma} .
$$

If we suppose $K^{*} \geq K_{L}$ or equivalently

$$
s_{e}^{*} \geq s_{e, L},
$$

we know that equation $(B .15)$ has a unique admissible solution $P_{1}\left(P^{*}\right)$ so that we are left with the system

$$
\left\{\begin{array}{l}
v^{*}=v_{L}-\Phi_{L}\left(P^{*}\right) \\
v^{*}=v_{R}+\Phi_{R}\left(P^{*}\right)
\end{array}\right.
$$

This system has a unique solution as soon as (B.13) holds or we allow vacuum to appear. Therefore, under the assumption (B.14), for any given $s_{e}^{*} \geq s_{e, L}$, the Riemann problem (7.10) has a unique self-similar solution.

\section{REFERENCES}

[1] R. Abgrall and S. Karni, Computations of compressible multifluids. J. Comput. Phys. 169 (2001) 594-623.

[2] J.J. Adimurthi and G.D. Veerappa Gowda, Godunov-type methods for conservation laws with a flux function discontinuous in space. SIAM J. Numer. Anal. 42 (2004) 179-208.

[3] E. Audusse and B. Perthame, Uniqueness for a scalar conservation law with discontinuous flux via adapted entropies, Inria research report No. 5261 (2004), France.

[4] D. Bale, R. LeVeque, S. Mitran and J. Rossmanith, A wave propagation method for conservation laws and balance laws with spatially varying flux functions. SIAM J. Sci. Comput. 24 (2002) 955-978.

[5] T. Barberon, Modélisation mathématique et numérique de la cavitation dans les écoulements multiphasiques compressibles. Thesis, University of Toulon, France (2002).

[6] F. Coquel, E. Godlewski, P.-A. Raviart et al., Numerical coupling of models in the context of fluid flows, work in preparation.

[7] S. Cordier, Hyperbolicity of the hydrodynamic model of plasmas under the quasi-neutrality hypothesis. Math. Methods Appl. Sci. 18 (1995) 627-647.

[8] B. Després, Lagrangian systems of conservation laws. Invariance properties of Lagrangian systems of conservation laws, approximate Riemann solvers and the entropy condition. Numer. Math. 89 (2001) 99-134.

[9] S. Diehl, On scalar conservation laws with point source and discontinuous flux function. SIAM J. Numer. Anal. 26 (1995) $1425-1451$.

[10] F. Dubois and P. Le Floch, Boundary conditions for nonlinear hyperbolic systems of conservation laws. J. Differential Equations 71 (1988) 93-122.

[11] R. Fedkiw, T. Aslam, B. Merriman and S. Osher, A non-oscillatory Eulerian approach to interfaces in multimaterial flows (the ghost fluid method). J. Comput. Phys. 152 (1999) 457-492.

[12] G. Gallice, Positive and entropy stable Godunov-type schemes for gas dynamics and MHD equations in Lagrangian or Eulerian coordinates. Numer. Math. 94 (2003) 673-713.

[13] M. Gisclon, Étude des conditions aux limites pour un système strictement hyperbolique via l'approximation parabolique. J. Math. Pures Appl. 75 (1996) 485-508.

[14] M. Gisclon and D. Serre, Étude des conditions aux limites pour un système hyperbolique, via l'approximation parabolique. C. R. Acad. Sci. Paris, Série I 319 (1994) 377-382.

[15] M. Gisclon and D. Serre, Conditions aux limites pour un système strictement hyperbolique fournies par le schéma de Godunov. RAIRO Modél. Math. Anal. Numér. 31 (1997) 359-380.

[16] E. Godlewski and P.-A. Raviart, Numerical approximation of hyperbolic systems of conservation laws. Appl. Math. Sci. 118, Springer, New York (1996).

[17] E. Godlewski and P.-A. Raviart, The numerical coupling of nonlinear hyperbolic systems of conservation laws: I. The scalar case. Numer. Math. 97 (2004) 81-130.

[18] M. Göz and C.-D. Munz, Approximate Riemann solvers for fluid flow with material interfaces. Numerical methods for wave propagation (Manchester, 1995), Kluwer Acad. Publ., Dordrecht. Fluid Mech. Appl. 47 (1998) 211-235.

[19] J.M. Greenberg, A.Y. Leroux, R. Baraille and A. Noussair, Analysis and approximation of conservation laws with source terms. SIAM J. Numer. Anal. 34 (1997) 1980-2007.

[20] A. Harten, P.D. Lax and B. van Leer, On upstream differencing and Godunov-type schemes for hyperbolic conservation laws. SIAM Rev. 25 (1983) 35-61.

[21] E. Isaacson and B. Temple, Nonlinear resonance in systems of conservation laws. SIAM J. Appl. Math. 52 (1992) $1260-1278$. 
[22] K. Karlsen, N. Risebro and J. Towers, Upwind difference approximations for degenerate parabolic convection-diffusion equations with a discontinuous coefficient. IMA J. Numer. Anal. 22 (2002) 623-664.

[23] R. Klausen and N. Risebro, Stability of conservation laws with discontinuous coefficients. J. Differential Equations 157 (1999) $41-60$.

[24] C. Klingenberg and N.H. Risebro, Stability of a resonant system of conservation laws modeling polymer flow with gravitation, J. Differential Equations 170 (2001) 344-380.

[25] S. Kokh, Aspects numériques et théoriques de la modélisation des écoulements diphasiques compressibles par des méthodes de capture d'interface. Thesis, University Paris 6, France (2001).

[26] K.-C. Le Thanh and P.-A. Raviart, Un modèle de plasma partiellement ionisé. Rapport CEA-R-6036, France (2003).

[27] W.K. Lyons, Conservation laws with sharp inhomogeneities. Quart. Appl. Math. 40 (1983) 385-393.

[28] S. Mishra, Convergence of upwind finite difference schemes for a scalar conservation law with indefinite discontinuities in the flux function. Ntnu Preprints on Conservation Laws 2003-077 (2003).

[29] C.-D. Munz, On Godunov-type schemes for Lagrangian gas dynamics. SIAM J. Numer. Anal. (1994), 17-42.

[30] T. Pougeard Dulimbert, Extraction de faisceaux d'ions à partir de plasmas neutres: Modélisation et simulation numérique. Thesis, University Paris 6, France (2001).

[31] N. Seguin and J. Vovelle, Analysis and approximation of a scalar conservation law with a flux function with discontinuous coefficients. Math. Models Methods Appl. Sci. 13 (2003) 221-257.

[32] D. Serre, Systèmes de lois de conservation I and II. Diderot éditeur, Paris (1996).

[33] J. Towers, A difference scheme for conservation laws with a discontinuous flux: the nonconvex case. SIAM J. Numer. Anal. 39 (2001) 1197-1218.

[34] Y.B. Zel'dovich and Y.P. Raizer, Physics of shock waves and high-temperature hydrodynamic phenomena, Vol. II. Academic Press (1967).

To access this journal online: www.edpsciences.org 